\title{
High Gain, Fast Scan, Broad Spectrum, Parallel Beam Wavelength Dispersive Spectrometer for SEM
}

\author{
Contract\# DE-FG02-02ER83545
}

\author{
Parallax Research, Inc.
}

September 11, 2003

\section{Summary}

On this six-month Phase I project, Parallax Research, Inc. investigated the feasibility of developing a new type of $\mathrm{x}$-ray spectrometer that would have very high energy resolution for the low energy $\mathrm{x}$-ray regime. This spectrometer would make use of previous technology developed by Parallax Research, Inc. for use in their Low Energy Xray Spectrometer (LEXS) but would extend the energy range to the entire $100-5000 \mathrm{eV}$ range enabling all elements to be detected with low energy excitation. Parallax proposed to:

Design new two-reflection $x$-ray optics to extend the energy range of the spectrometer.

Fabricate an Interim Test Optic to demonstrate the fabrication technology.

Test the Interim Optic

Design a full energy range spectrometer for Phase II.

Parallax Research, Inc. was successful on all of these tasks with the exception of completely testing the interim optic but was able to do some testing. An analytical solution for the two-reflection optics was derived for Task 1. The Interim Test Optic was fabricated and assembled. We partially tested the new experimental diffraction optic and built a test fixture for the Interim Optic. We completed the conceptual design of the full spectrometer to be assembled and tested in Phase II.

To achieve good analytical spatial resolution in Scanning Electron Microscopes (SEMs), they are frequently being used at very low beam voltages $(<10 \mathrm{KV})$ that limits their ability to excite $\mathrm{x}$-ray lines from higher $\mathrm{Z}$ elements. With this new high-resolution spectrometer, it will be possible to use a combination of $\mathrm{K}, \mathrm{L}$, and $\mathrm{M}$ lines to detect all of the elements in the periodic table with great accuracy. 


\title{
High Gain, Fast Scan, Broad Spectrum, Parallel Beam Wavelength Dispersive Spectrometer for SEM
}

Parallax Research, Inc.

\author{
David OHara \& Dr. Eric Lochner
}

\section{Identification of the Problem and Opportunity}

In electron beam microanalysis, there are two major types of $\mathrm{x}$-ray elemental analytical instruments, EDS (Energy Dispersive Spectrometers) and WDS (Wavelength Dispersive Spectrometers) with EDS being the far more common variety. EDS spectrometers use a crystal in which an incident $x$-ray produces pulses of current whose voltage is a measure of the x-ray energy. WDS spectrometers use crystals in which Bragg diffraction is used to select $\mathrm{X}$-ray wavelengths where the $\mathrm{x}$-rays are then counted by a detector. WDS was the first spectrometer type used for electron beam microanalysis but has been supplanted by EDS due to ease of use relative to WDS. However, because WDS offers far better energy resolution relative to EDS, it is used when there are peak overlaps such as the $\mathrm{Pb}(\mathrm{M})$ and $\mathrm{S}(\mathrm{K})$ or $\mathrm{N}(\mathrm{K})$ and $\mathrm{Ti}(\mathrm{L})$ lines. Conventional WDS is a slow technique where each elemental line is slowly sequentially scanned one after another and it may take up to an hour to do a full elemental scan. By contrast, EDS measures all elements simultaneously, although at lower resolution than WDS. A modern EDS may have about $129 \mathrm{eV}$ resolution at the Mn line while a WDS system can have a resolution of $8 \mathrm{eV}$. A spectrometer that had the resolution of WDS but was able to do a full scan as quickly as an EDS would revolutionize x-ray spectrometry for electron beam microanalysis. Parallax Research, Inc. proposes to develop such a spectrometer based on the successful Phase I research.

Over the past few years, micro-calorimeter detectors have been developed which seem to offer the desired features but they have a few problems. They seem to be limited to fairly low count rates and are very expensive with a model recently introduced by a spectrometer manufacturer costing over $\$ 400,000$, more than most of the electron microscopes on which it might be used. Even a low cost EDS system can have count rates of over 20,000 cps and WDS systems can have over 500,000 cps so the count rate limitations of the micro-calorimeters are a problem.

Parallax Research, Inc. has introduced a lower cost spectrometer that partially fills the requirements. The LEXS (Low Energy X-ray Spectrometer) is a fast scanning WDS spectrometer optimized for the low energy region where there are many peak overlaps. LEXS can count up to 1,000,000 cps in some cases and is priced lower than a conventional full range WDS. LEXS can scan over the 100-2400 eV range fairly fast so that by looking only at the major spectral peaks in that range it can sometimes do scans as fast as an EDS spectrometer covering the range up to $10,000 \mathrm{eV}$. LEXS has the advantage of being unusually sensitive to the very low energy range where EDS has 
problems and where there are many overlaps. LEXS still suffers from a flaw that prevents it from replacing EDS spectrometers in some applications; it only covers the range of 100-2400 eV that means that it does not detect some elements of interest such as $\mathrm{Ba}, \mathrm{Cd}$, In and Te. In general, LEXS does not easily detect elements of atomic \#43-56 and $\mathrm{K}$ and $\mathrm{Bi}$. LEXS must be used with an EDS detector to achieve full spectral coverage. Clearly, what is needed is a spectrometer that incorporates the concepts of the LEXS spectrometer but covers a much larger spectral range.

In this program we propose to produce such a spectrometer which may be used to replace EDS spectrometers in some applications giving very good spectral resolution, high count rate capability, ease of mounting on various electron beam instruments, and the sensitivity of the LEXS spectrometer to low X-ray energies. Like LEXS, it will be a Parallel Beam Spectrometer that does not have a Rowland Circle but instead uses an $\mathrm{x}$-ray collimation optic to redirect $\mathrm{x}$-rays diverging from the source into a parallel beam that is incident on a flat diffractor but it will not have the energy limitations of LEXS. The solid angle of emitted x-rays collected by the optics in LEXS drops very rapidly with increasing energy so that by the time $2400 \mathrm{eV}$ is reached, the performance relative to a conventional WDS is very poor. We propose to extend this energy range out to the $\mathrm{Ti}(\mathrm{K})$ line (and possibly out to the $\mathrm{Fe}(\mathrm{K})$ line at $6400 \mathrm{eV}$ ) so that the resulting spectrometer can cover the entire periodic table using a combination of $\mathrm{K}, \mathrm{L}, \mathrm{M}$, and $\mathrm{N}$ lines. If this spectrometer can scan fast enough, it may be able to replace EDS systems for some applications.

\section{Phase I Technical Objectives}

In this program, Parallax proposed to develop a new $\mathrm{x}$-ray spectrometer based on grazing incidence optics that collects a large solid angle of higher energy x-rays and then directs them onto a suitable diffractor. This optic will avoid the problem of the polycapillary optics by use of suitable reflecting surfaces and by achieving considerably better exit collimation than the polycapillary. This optics will utilize two grazing incidence reflections at smaller angles than the existing grazing incidence optics thus allowing higher energies to be collected. Previously, Parallax Research has not fabricated two-reflection grazing incidence optics due to the perceived difficulty, risk of failure and expense. Although Parallax had developed the technology for making the single reflection $x$-ray optics, we never felt we had developed it sufficiently to make the more complex two-reflection x-ray optics. Now, with considerably more experience, we think we can fabricate such optics with diminished risk. Using this two-reflection optics, we proposed to design a full spectrometer for the $100-4500 \mathrm{eV}$ spectral region. Parallax Research, Inc. proposed the following tasks for this effort:

1. Design collimation optics. This optics will be either two-reflection optics utilizing combinations of hyperboloidal and paraboloidal surfaces or a combination of paraboloid-like and conical surfaces arranged to produce a wellcollimated beam from the exit aperture. The optics will consist of multiply nested reflectors. 
2. Fabricate an interim test optic to develop the technology for producing the small nested optics to be made in Phase II.

3. Test the interim optic using a combination of a PET and experimental graphite diffractor.

4. Produce a preliminary design for a full spectrometer to be built in phase II.

Task 1.

Design collimation optics.

Both the Parallax Research, Inc. Low Energy X-ray Spectrometer (LEXS) and the NORAN MaXRay spectrometer are examples of Parallel Beam WDS X-ray spectrometers that do not use Rowland Circle geometry. Instead they use an X-ray collection/collimation optic to collect the x-rays diverging from the source and then to redirect them into a fairly parallel beam that is then incident on a flat diffractor. This geometry has advantages of collecting a very large solid angle of low energy x-rays and the use of flat diffractors which produces very high count rates for low energy x-rays. Using this geometry, the LEXS spectrometer is able to achieve count rates and Peak to Background ratios (P/B) for energies below $1 \mathrm{KeV}$ much higher than conventional spectrometers.

LEXS uses a collimating optic that consists of three confocal nested grazing incidence paraboloids to collect the diverging x-rays. The outermost paraboloid has large grazing angles for the energies below $800 \mathrm{eV}$ and has a reflecting surface of Ni while the two inner paraboloids have successively smaller grazing angles and have gold reflecting surfaces for higher energies. The NORAN MaXRay spectrometer also uses similar optics made by Parallax Research, Inc. These optics work very well for energies below about $1250 \mathrm{eV}$ but the available grazing angle drops very rapidly with increasing $\mathrm{x}$-ray energy so that by the time x-rays are at $2400 \mathrm{eV}$, they do not perform well. At the 525 eV Oxygen line, the optics half collection angle is 13 degrees for a solid collection angle of .16sr while at $2400 \mathrm{eV}$ it is only a 3-degree half cone angle for a solid angle of .0086 sr.

NORAN Instruments, Inc. MaXRay spectrometer used the original Parallax collimation optics that had sufficient surface roughness that a considerable fraction of the $\mathrm{x}$-rays at 1500-2400 eV was poorly collimated so the count rates were poor for these energies. For this reason, NORAN chose to use polycapillary collimation optics made by X-ray Optical Systems combined with parts of the Parallax optics to extend the range of their optics. The resulting spectrometer; the MaXRay ER has performance out to 10,000 $\mathrm{eV}$ but around the interesting $\mathrm{Zr} / \mathrm{P}$ overlap at $2000 \mathrm{eV}$, it has very poor performance and the performance is not very good even at higher energies. This poor performance around $2000 \mathrm{eV}$ is due to the Si absorption edge in the polycapillary optics and the poor performance at higher energies is due to the poor collimation of the x-rays by the polycapillary optic. At energies between the Si absorption edge at $1840 \mathrm{eV}$ and about $2500 \mathrm{eV}$, the MAXRay ER has very poor performance due to the Si absorption edge of the glass capillaries. 
At higher x-ray energies, the so-called critical angle for total reflection is very sharp where at lower energies it is "smeared" by absorption so that at angles less than the critical value, the reflectivity may be considerably less than unity and at angles greater than the critical value it may be greater than zero. At higher energies (say, above 2000 $\mathrm{eV}$ ) where the critical angle is fairly sharp, reflection optics can be used with several reflections so the losses in each reflection are low. This is how the polycapillary optics work with each reflection being at a very small angle and the optic uses 6-12 reflections to bend the $\mathrm{x}$-rays through the desired angle. The same polycapillary optics do not work well at very low energies because of the losses due to absorption. At energies starting around $1500 \mathrm{eV}$, a two-reflection grazing incidence optic may be used to collimate $\mathrm{x}$-rays with greater efficiency than a single reflection collimator if we insure that the reflection angles are at less than the critical angle. By using two reflections, we can extend the performance of the collimator in a parallel beam spectrometer to higher energies while avoiding the problems of the polycapillary optic.

Various geometries of two reflection collimators will work to redirect $\mathrm{x}$-rays emanating from a point source into a parallel beam including combinations of hyperbola/parabola and parabola-like/cone geometries and we have based our calculations on the so-called Wolter I geometry of a hyperbola followed by a parabola. This is really just a Wolter I X-ray telescope used in reverse except the Wolter I design for astronomical $\mathrm{x}$-ray telescopes is used to minimize aberrations found in grazing incidence optics while we just use it to capture a larger solid angle than a single reflection optic. The Wolter I geometry is illustrated in Fig. 2. showing that the hyperboloidal surface and paraboloidal surfaces have common foci with all reflections being at angles smaller than the critical angle. The Wolter 1 design used for our calculation consists of 5 nested Wolter 1 reflecting shells with the outer ones reflecting the lower energies. Other geometries are possible for a two reflection collimating optic and in Phase I we will choose the most efficacious in terms of ease of manufacture and collimation ability.

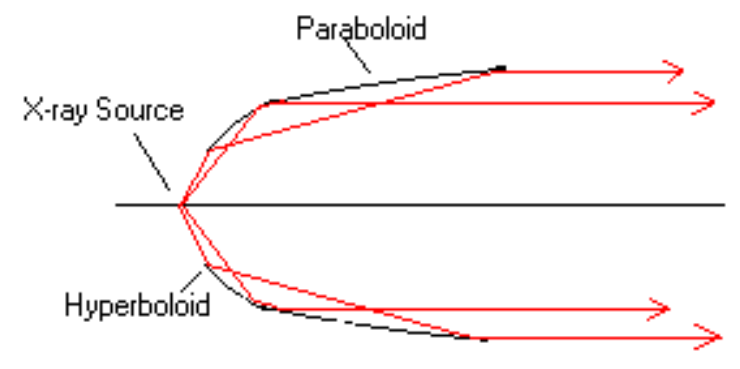

Fig 2. Wolter $1 \mathrm{x}$-ray collimator.

To adequately assess the design of the Phase II optic, it was necessary to fully develop a model of both the previous LEXS optic and the new "NexGen" optic and this task was assigned to Dr. Eric Lochner. His report of the performance of the previous optic and design of the two-reflection NexGen optics is included here. 


\section{LEXS Reflective Optic Design: Theory}

\section{Overview}

The existing LEXS reflective optic consists of three coaxially nested paraboloids of revolution. Having a common focus, these paraboloids act as collimators for X-rays produced from a point source. The optic can be considered as a set of paraboloidal surfaces

$$
\left\{\Sigma_{i}\right\}
$$

where each surface, $\Sigma_{i}$, is a paraboloid of revolution defined as

$$
\rho^{2}=4 \alpha_{i}\left(z+a_{i}\right)
$$

$z$ is the axis of rotation, while $\alpha_{i}$ and $a_{i}$ are parameters defining the shape of the paraboloid: $\alpha_{i}$ defines the curvature of the paraboloid, while $a_{i}$ determines where the vertex is relative to the origin. For the existing LEXS reflective optic, $i=3$, while for the NextGen extended range reflective optic $i=4$. The design process is the problem of determining the sets of parameters

$$
\left\{\alpha_{i}\right\},\left\{a_{i}\right\}
$$

from design considerations. For the LEXS reflective optic, this constitutes up to six parameters and for the NextGen eight parameters.

\section{$\underline{\text { Design Parameters }}$}

The design of an optic appropriate to a specified energy range demands a consideration of the reflectivity of the interior surface of a paraboloid over its range of grazing angles. The outer paraboloid, $\Sigma_{l}$, will involve the largest grazing angles, and thus is the optic primarily responsible for the collimation of lower energies. Involving the smallest range of grazing angles, the inner paraboloid, $\Sigma_{3}$, is thus primarily responsible for the collimation of the highest energies. Given the reflectivity as a function of grazing angle for a selected reflective coating material, the maximum grazing angle, $\gamma_{\max }$, at the entrance of the outer paraboloid is specified as a design parameter (See Figure 1.1). This will determine the minimum reflectivity achieved on the outer paraboloidal surface. In principle, $\gamma_{\max }$ is determined by defining an arbitrary threshold to the reflectivity. The material aspect of LEXS optic design, including reflective coating selection and the roughness of the reflective surface, will be addressed below. Only the design aspects related to mandrel design are currently addressed in this section. 


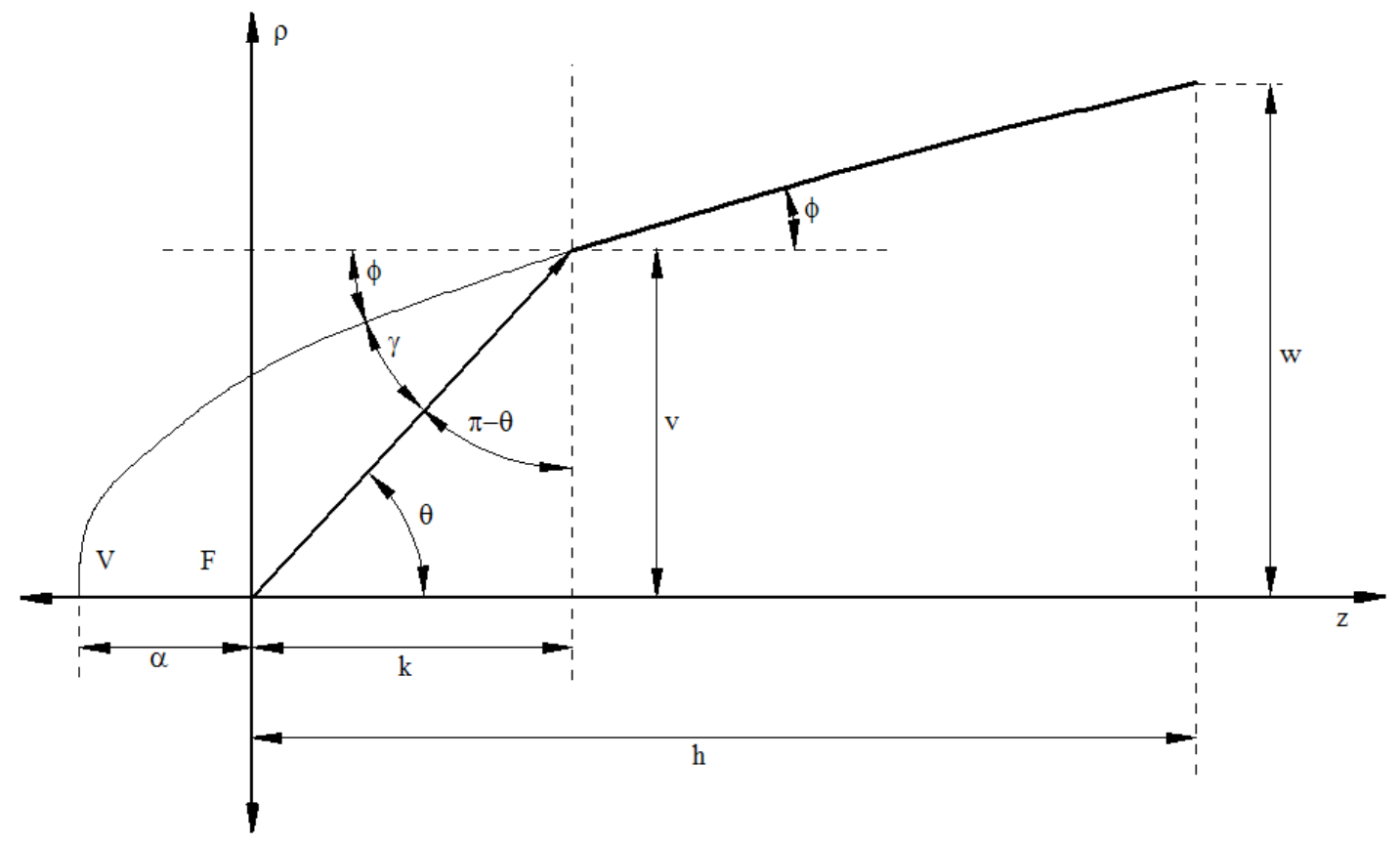

Figure 1.1. The parameters $\gamma_{\max }, k \& v, h \& w$ involved in the LEXS optic design. $V$ is the vertex of the paraboloid. The origin of coordinates has been shifted to the focus, $F$.

Another design consideration is the solid angle accepted by the optic. Ideally the solid angle should be chosen to be as large as possible given the physical constraints of the application. Two design parameters are $k$, the distance between the focus and the front of the set of nested paraboloids, and $v_{l}$, the radius of the front end of the first, or outermost, paraboloid, $\Sigma_{l}$. The solid angle, $\Omega_{l}$, subtended by the front of the outermost paraboloid $\Sigma_{l}$ is:

$$
\Omega_{1}=2 \pi\left(1-\cos \theta_{\text {max }, 1}\right)
$$

where $\theta_{\max , 1}$ is the angle subtended by the entrance of the outermost paraboloid $\Sigma_{l}$. From trigonometry, the $\cos \theta_{\max , 1}$ is defined as:

$$
\cos \theta_{\max , 1}=\frac{k}{\sqrt{k^{2}+v_{1}^{2}}}
$$

which can be input into Equation 1.4 to provide

$$
\Omega_{1}=2 \pi\left(1-\frac{k}{\sqrt{k^{2}+v_{1}^{2}}}\right) .
$$


Equation 1.6 can be solved for $v_{l}$ :

$$
v_{1}=k \sqrt{\frac{1}{\left(1-\frac{\Omega_{1}}{2 \pi}\right)^{2}}-1} .
$$

Given $\Omega_{l}$ and $k, v_{l}$ can be obtained, or alternately, given $k$ and $v_{l}, \Omega_{l}$ can be obtained. In the present work, $\gamma_{\max }, k$ and $v_{l}$ will be considered the "fundamental" design parameters, and will be used to calculate $\alpha_{l}$. This will be demonstrated below.

The last design parameter is that the entire optic be of length $l$. If $k$ is the distance between the focus and the front of the optic, and $h$ is the distance between the focus and the end of the optic, then

$$
l=h-k
$$

The radius of the outermost paraboloid $\Sigma_{1}$, can be obtained from Equation 1.2:

$$
w^{2}=4 \alpha_{1}\left(h+a_{1}\right)
$$

While $w$ may not seem important to the LEXS reflective optic design, it is important in calculating the input radius, $v_{2}$, of the middle optic, $\Sigma_{2}$, due to the added constraint that a reflecting surface not block the solid angle accepted by its immediately exterior reflecting surface. Thus $\Sigma_{3}$ may not block the solid angle accepted by $\Sigma_{2}$, and $\Sigma_{2}$ may not block the solid angle accepted by $\Sigma_{l}$. This too will be outlined below.

\section{$\underline{\text { Normalizing Coordinates }}$}

To facilitate ease in representing the paraboloids, the coordinates were shifted to place the origin at the focus, F. This defines the parameters $a_{i}=\alpha_{i}$ since the distance between the vertex, $\mathrm{V}$, and the focus $\mathrm{F}$, for surface $\Sigma_{i}$ is $\alpha_{i}$. In addition to the pragmatic reasons for placing the focus at the origin, it simplifies subsequent calculations by allowing each paraboloid to be defined by only one parameter, $\alpha$. Thus, in the remainder of this discussion, the following form for the paraboloids will be utilized:

$$
\rho^{2}=4 \alpha_{i}\left(z+\alpha_{i}\right)
$$




\section{Calculating the Angles}

For a given surface, $\Sigma_{i}$, the slope angle, $\phi$, of a ray intersecting the interior surface can be calculated from the derivative of Equation 1.10. See Figure 1.1. The slope, $m$, is equal to $\tan \phi$, so

$$
m=\tan \phi=\frac{d \rho}{d z}=\sqrt{\frac{\alpha_{i}}{z+\alpha_{i}}} .
$$

The angle of a ray intersecting the interior surface of $\Sigma_{i}$ and the $z$-axis, $\theta$, is

$$
\tan \theta=\frac{\rho}{z}=\frac{2 \sqrt{\alpha_{i}\left(z+\alpha_{i}\right)}}{z} .
$$

The grazing angle, $\gamma$, can be calculated using from $\theta$ and $\phi$

$$
\gamma=\theta-\phi
$$

Inserting Equations 1.11 and 1.12 into 1.13 this yields

$$
\gamma=\tan ^{-1} \sqrt{\frac{\alpha_{i}}{z+\alpha_{i}}}+\tan ^{-1} \frac{2 \sqrt{\alpha_{i}\left(z+\alpha_{i}\right)}}{z}
$$

The tangent of the grazing angle is expressible as

$$
\tan \gamma=\tan (\theta-\phi)=\frac{\tan \theta-\tan \phi}{1+\tan \theta \tan \phi}=\frac{\frac{\rho}{z}-\sqrt{\frac{\alpha_{i}}{z+\alpha_{i}}}}{1+\frac{\rho}{z} \sqrt{\frac{\alpha_{i}}{z+\alpha_{i}}}}
$$

These relationships define the angles $\gamma, \theta$ and $\phi$, as well as their tangents. They will be used extensively in the bulk of this work.

\section{$\underline{\text { Calculating }} \alpha_{1}$ from Design Parameters}

The design parameter $\gamma_{\max }$ allows us to determine the parameter $\alpha_{1}$ which defines the shape of the outer paraboloid $\Sigma_{l} \cdot \gamma_{\max }$ occurs at the front end of $\Sigma_{l}$ so that $\tan \gamma_{\max }$ can be defined by Equation 1.15 by setting $\rho=v_{1}$ and $z=k$ 


$$
\tan \gamma_{\max }=\frac{\frac{v_{1}}{k}-\sqrt{\frac{\alpha_{1}}{k+\alpha_{1}}}}{1+\frac{v_{1}}{k} \sqrt{\frac{\alpha_{1}}{k+\alpha_{1}}}}
$$

which can be solved for $\alpha_{1}$ :

$$
\frac{1}{\alpha_{1}}=\frac{1}{k}\left(\left(\frac{k+v_{1} \tan \gamma_{\max }}{v_{1}-k \tan \gamma_{\max }}\right)^{2}-1\right)
$$

$\alpha_{1}$ is thus $\alpha_{1}\left(\gamma_{\max }, k, v_{1}\right)$, a function of the design parameters that define the front end of the surface $\Sigma_{1}$. $\alpha_{1}$ can thus be used to determine the radius of the back end of the paraboloid $\Sigma_{l}$ using Equation 1.10 by setting $z=h$ :

$$
w_{1}^{2}=w_{1}^{2}\left(\alpha_{1}\left(\gamma_{\max }, k, v_{1}\right), h\right)=4 \alpha_{1}\left(\gamma_{\max }, k, v_{1}\right)\left(h+\alpha_{1}\left(\gamma_{\max }, k, v_{1}\right)\right)
$$

$\underline{\mathrm{Using}} \alpha_{1} \underline{\text { to Solve for } \alpha_{i} \text { of Interior Paraboloids }}$

The above determination of $\alpha_{1}$ and $w_{1}$ from the design parameters $\left(\gamma_{\max }, k\right.$ and $v_{1}$ provides sufficient information to determine $\alpha_{2}$ of the next inner paraboloid $\Sigma_{2}$. As discussed above, an additional design constraint is that the nested paraboloids do not block each other's accepted solid angle. Thus, $\Sigma_{2}$ must be of such a shape as to not block any of the reflective surface of $\Sigma_{l}$. See Figure 1.2.

Ranges of grazing angle $\gamma$, and acceptance angle, $\theta$, for surface $\Sigma_{1}$ are defined by the ray extrema which originate from the focus (origin) and intersect the far and near edges of the paraboloidal section. These ranges will eventually be calculated for the final set of design parameters for the LEXS optic, as they will be required to integrate inner surface reflectivities over the surface areas of each paraboloid.

For surface $\Sigma_{2}$ to not block rays from intersecting the surface $\Sigma_{1}$, the surface $\Sigma_{2}$ must be within the cone defined by $\theta_{\text {min,l }}$. For $\Sigma_{2}$ to simultaneously have the maximum acceptance solid angle, the paraboloidal surface $\Sigma_{2}$ must intersect the cone of angle $\theta_{\min , 1}$ at $z$ $=k$.

The ray from the focus to the back edge of surface $\Sigma_{l}$ has slope

$$
m=\tan \theta_{\min , 1}=\frac{w_{1}}{h}=\frac{2 \sqrt{\alpha_{1}\left(\gamma_{\max }, k, v_{1}\right)\left(h+\alpha_{1}\left(\gamma_{\max }, k, v_{1}\right)\right)}}{h}
$$




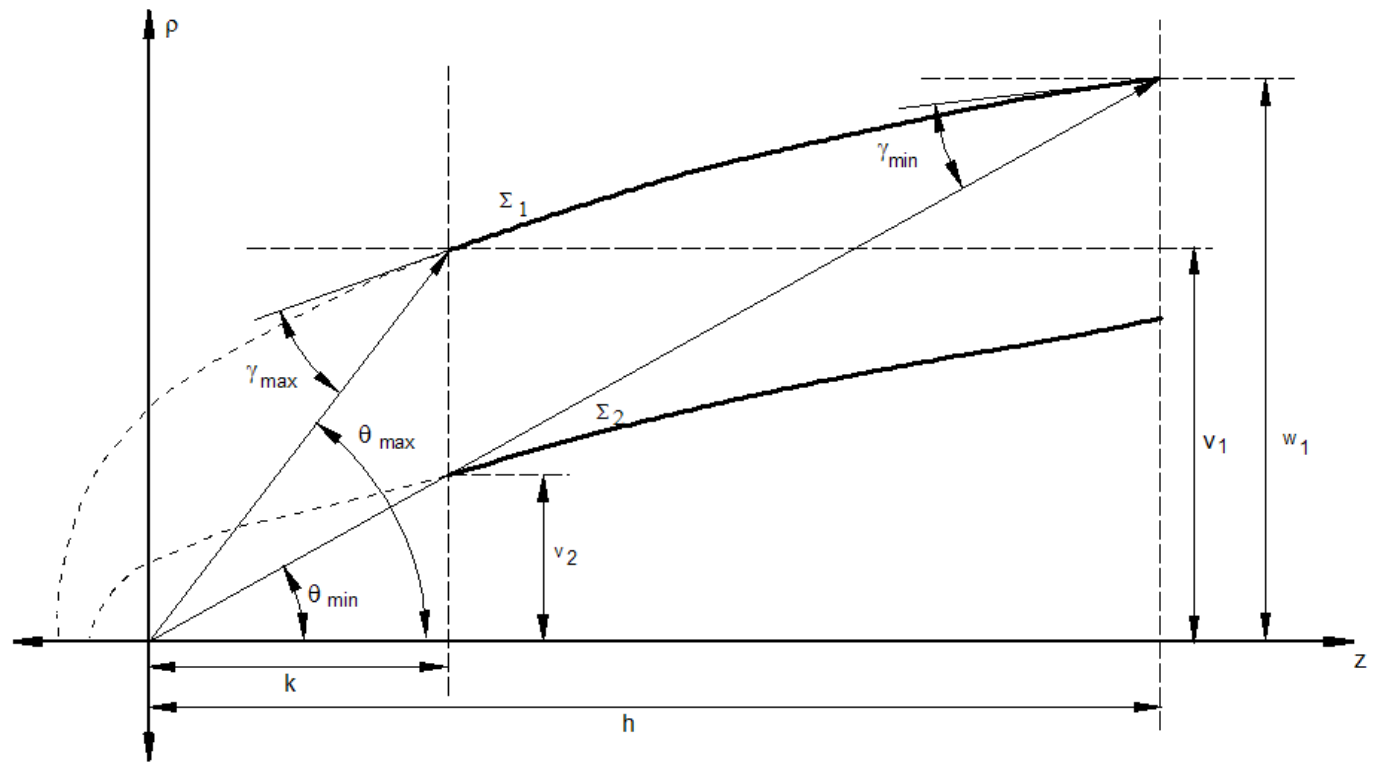

Figure 1.2. This figure shows the constraint that a nested interior reflective surface not block the reflective surface of the immediately exterior surface.

and thus can be represented by the equation

$$
\rho=m z=\frac{w_{1}}{h} z=\frac{2 \sqrt{\alpha_{1}\left(\gamma_{\max }, k, v_{1}\right)\left(h+\alpha_{1}\left(\gamma_{\max }, k, v_{1}\right)\right)}}{h} z
$$

If the equation for surface $\Sigma_{2}$ is

$$
\rho^{2}=4 \alpha_{2}\left(z+\alpha_{2}\right)
$$

then substituting Equation 1.20 into Equation 1.21

$$
\frac{w_{1}^{2}}{h^{2}} z^{2}=4 \alpha_{2}\left(z+\alpha_{2}\right)
$$

As the front of the optic is defined by $z=k$,

$$
\frac{w_{1}^{2} k^{2}}{h^{2}}=k^{2} \tan ^{2} \theta_{\min , 1}=4 \alpha_{2}\left(k+\alpha_{2}\right)
$$

which can be written as a quadratic equation

$$
4 \alpha_{2}^{2}+4 k \alpha_{2}-\frac{w_{1}^{2} k^{2}}{h^{2}}=0
$$


and taking the positive root, solved for $\alpha_{2}$ :

$$
\alpha_{2}=\frac{k \sqrt{h^{2}+w_{1}^{2}}-h}{2 h} \text {. }
$$

Inserting the full form of $w_{1}$ from Equation 1.18

$$
\alpha_{2}=\frac{k \sqrt{h^{2}+2 \sqrt{\alpha_{1}\left(\gamma_{\max }, k, v_{1}\right)\left(h+\alpha_{1}\left(\gamma_{\max }, k, v_{1}\right)\right)}}-h}{2 h}
$$

it becomes evident that $\alpha_{2}=\alpha_{2}\left(h, k, \alpha_{1}\right)=\alpha_{2}\left(\gamma_{\max }, k, h, v_{1}\right)$.

Having found $\alpha_{2}$, which defines the shape of the inner reflecting surface $\Sigma_{2}$, the above method can be repeated to find $\alpha_{3}$ which defines the shape of the innermost reflecting surface $\Sigma_{3}$. $\alpha_{3}$ will subsequently be a function of $h, k$ and $\alpha_{2}$, and thus ultimately of $\gamma_{\max }, h, k$ and $v_{l}$. Thus, the three nested paraboloids are defined solely by the parameters: $\gamma_{\max }, h, k$ and $v_{l}$.

\section{LEXS Reflective Optic Design: Geometric Results}

The methodology described above in Section I, was used to define a set of three nested paraboloidal sections which forms the existing LEXS reflective X-ray optic. Because of the high reflectivity of $\mathrm{Ni}$ at low energies for grazing angles lower than $100 \mathrm{mrad}$, the outermost paraboloid, was designed around this material choice. Figure 1.3 below shows the reflectivity of a C Ka X-rays at $277 \mathrm{eV}$ from a Ni surface up to a grazing angle of $100 \mathrm{mrad}$. The reflectivity was calculated using the freeware program SF by Mark Thomas at Lawrence Berkeley National Laboratory. Given the atomic number, density and thickness of a reflector, SF allows the user to obtain either reflectivity as a function of X-ray energy at a fixed grazing angle, or reflectivity as a function of grazing angle at a fixed X-ray energy. An advantage of SF over calculating the reflectivities directly using Fresnel equations, is that it includes absorption at X-ray absorption edges, an effect that must be considered in the design of reflective optics. The data in Figure 1.3 was determined for pure Ni with a bulk density of $8.9 \mathrm{gcm}^{-3}$ and a thickness of $1000 \mu \mathrm{m}$. An arbitrary reflectivity cutoff of $50 \%$ was set, which corresponded to a grazing angle of roughly $110 \mathrm{mrad}$ or 6.25 degrees. This thus sets the design parameter $\gamma_{\max }$ :

$$
\gamma_{\max }=6.25 \text { degrees }=109.09 \mathrm{mrad}
$$

The distance between the focus and the front and back of the LEXS optic were set to practical values for an optic to be incorporated into an SEM for WDS applications. The length of the optic was set to approximately $6.5 \mathrm{~cm}$, with the distance between the focus 


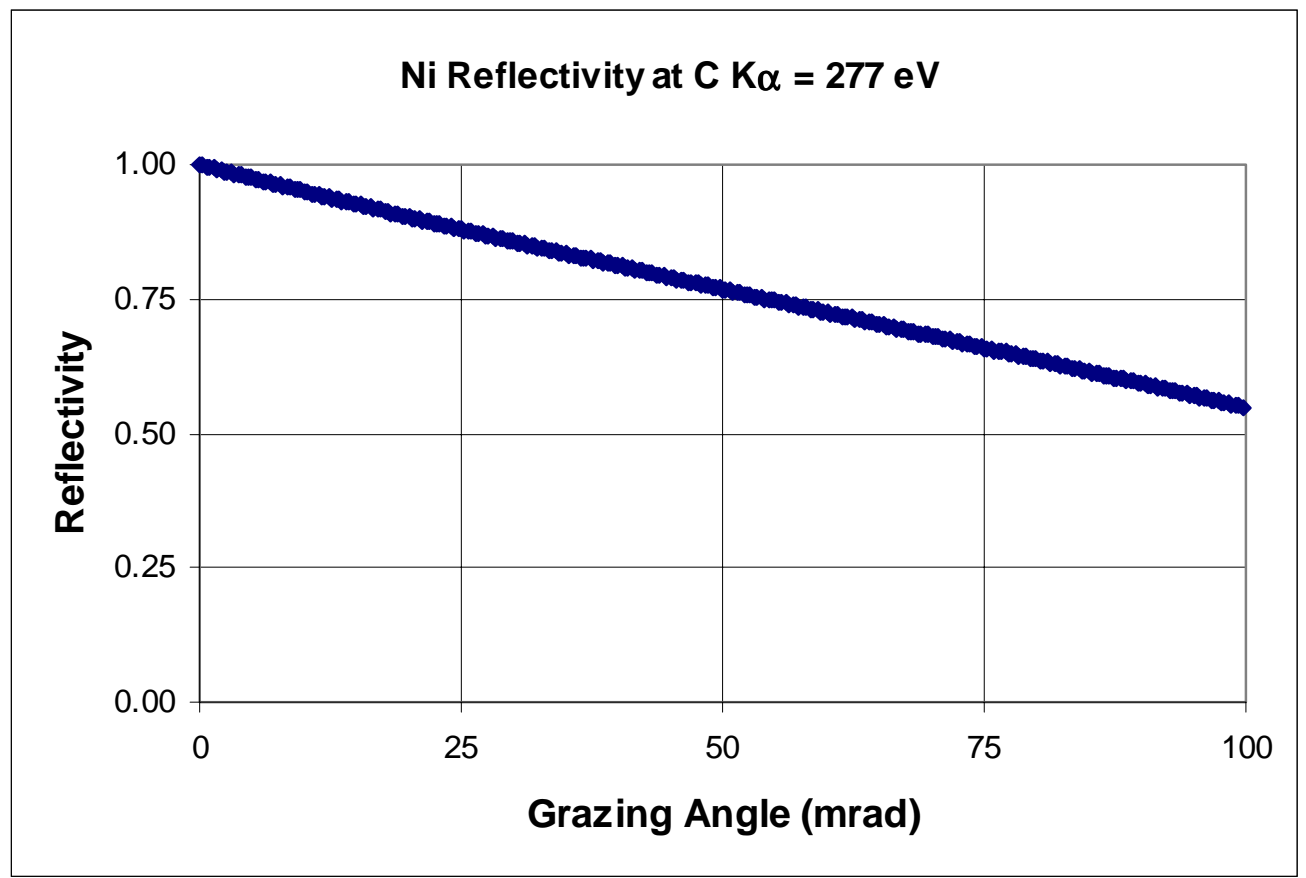

Figure 1.3. The reflectivity from $\mathrm{Ni}$ of $\mathrm{C} \mathrm{K}_{\alpha} \mathrm{X}$-rays at $277 \mathrm{eV}$ up to a grazing angle of $100 \mathrm{mrad}$.

and the front of the optic set to be $1.9888 \mathrm{~cm}$, and the distance between the focus and the back of the optic set to be $8.4888 \mathrm{~cm}$. Thus, $k$ and $h$ are fixed:

$$
\begin{aligned}
k & =1.9888 \mathrm{~cm} \\
h & =8.4888 \mathrm{~cm} .
\end{aligned}
$$

The outermost paraboloid was chosen to accept a solid angle of approximately 150 mster, corresponding to a $\theta_{\max , 1}$ of approximately 12.5 degrees, which can be used to determine a value of $v_{l}$ of

$$
\mathrm{v}_{1}=0.4356 \mathrm{~cm} \text {. }
$$

These parameters, $\gamma_{\max }, h, k$ and $v_{l}$, were then used to calculate the set of $\left\{\alpha_{i}\right\}$ using the formalism described above. The results are shown in Table 1.1:

\begin{tabular}{|c|c|}
\hline paraboloid & $\alpha$ \\
\hline$\Sigma_{1}$ (outer) & 0.023850 \\
\hline$\Sigma_{2}$ & 0.005550 \\
\hline$\Sigma_{3}$ (inner) & 0.012945 \\
\hline
\end{tabular}

Table 1.1. LEXS paraboloid parameters, $\alpha_{i}$. 
The ranges of grazing angle $\gamma$, and acceptance angle, $\theta$, are calculated for the paraboloids in Tables 1.2 and 1.3 below. These will be used extensively below in the integration of reflectivities over the paraboloidal surfaces.

\begin{tabular}{|c|c|c|}
\hline paraboloid & $\gamma_{\min }(\mathrm{mrad})$ & $\gamma_{\max }(\mathrm{mrad})$ \\
\hline$\Sigma_{1}$ (outer) & 52.95 & 109.09 \\
\hline$\Sigma_{2}$ & 25.59 & 52.90 \\
\hline$\Sigma_{3}$ (inner) & 12.37 & 25.65 \\
\hline
\end{tabular}

Table 1.2. LEXS ranges of grazing angles.

\begin{tabular}{|c|c|c|}
\hline paraboloid & $\theta_{\min }(\mathrm{mrad})$ & $\theta_{\max }(\mathrm{mrad})$ \\
\hline$\Sigma_{1}$ (outer) & 105.91 & 218.16 \\
\hline$\Sigma_{2}$ & 51.18 & 105.92 \\
\hline$\Sigma_{3}$ (inner) & 24.73 & 51.30 \\
\hline
\end{tabular}

Table 1.3. LEXS ranges of acceptance angles.

\section{LEXS Reflective 0ptic Design: Materials}

Given the above calculated ranges of grazing angle, the appropriate reflective materials must be chosen in order to optimize the paraboloid's effective reflectivity. The outer paraboloid, $\Sigma_{l}$, was selected above to be $\mathrm{Ni}$ in determining the maximum grazing angle, $\gamma_{\max }$, for a $50 \%$ reflectivity threshold. The reflective material of the other surfaces, $\Sigma_{2}$ and $\Sigma_{3}$, still require determination.

Having rigorously solved for the properties of the existing LEXS optics, Lochner then looked at various options for extending the energy range of these or similar optics.

\section{N extGen Design: Theoretical Basis}

\section{Overview}

Extending the performance of the LEXS parabolic reflective optic can be accomplished in three ways. The first is an increase of the reflectivity of the reflective surfaces. The choice of $\mathrm{Ni}$ and $\mathrm{Au}$ for the existing LEXS optic design was explained in Chapter 2. An exploration of alternative reflective materials could theoretically result in an increased integrated reflectivity of the surfaces defined in Chapter 1. Materials choices are, as explained in Chapter 2, motivated by not only the reflectivity and absorption edges of the 
reflective metals, but also the materials synthesis technologies. The second avenue for increasing the performance of the LEXS optic is increasing its collected solid angle. An arbitrary increase in collected solid angle is not necessarily an improvement, and may actually lead to a degradation of optic performance. If the increase of collected solid angle results in increased grazing angles, then the integrated reflectivities will decrease and the product, the total effective solid angle, will remain unchanged or may decline. Physically larger optics would also demand the scaling of the rest of the LEXS spectrometer: larger diffractors, chamber size and detector window. The last approach, and the one utilized here, is to reduce the maximum grazing angle, while neither increasing the optic size nor decreasing the collected solid angle, through the use of a two reflection optic. Thes three avenues towards extending the LEXS performance will be discussed in this chapter.

\section{Extending Reflectivity: An Analysis of Options}

Figure 3.1 below shows the reflectivity of various refractory and precious metals of groups VIA, VIIA, VIIIA, and IB. The reflectivities were calculated using the program described in Chapter 2, SF, for bulk densities and reflective layer thicknesses of $1000 \mu \mathrm{m}$. The grazing angle for the data in Figure 3.1 is set to $12.4 \mathrm{mrad}$, which corresponds to the far end or the inner reflective surface, $\Sigma_{3}$. This portion of $\Sigma_{3}$ is most efficient at reflecting higher energy $\mathrm{X}$-rays due to the smaller grazing angle, and thus the energy range of 2000$6000 \mathrm{eV}$ is displayed to reflect this region of utility, and projected extended utility. Data displayed with hollow circles are row four elements, those displayed with solid diamonds are row five elements, and those with solid squares are row six elements. 


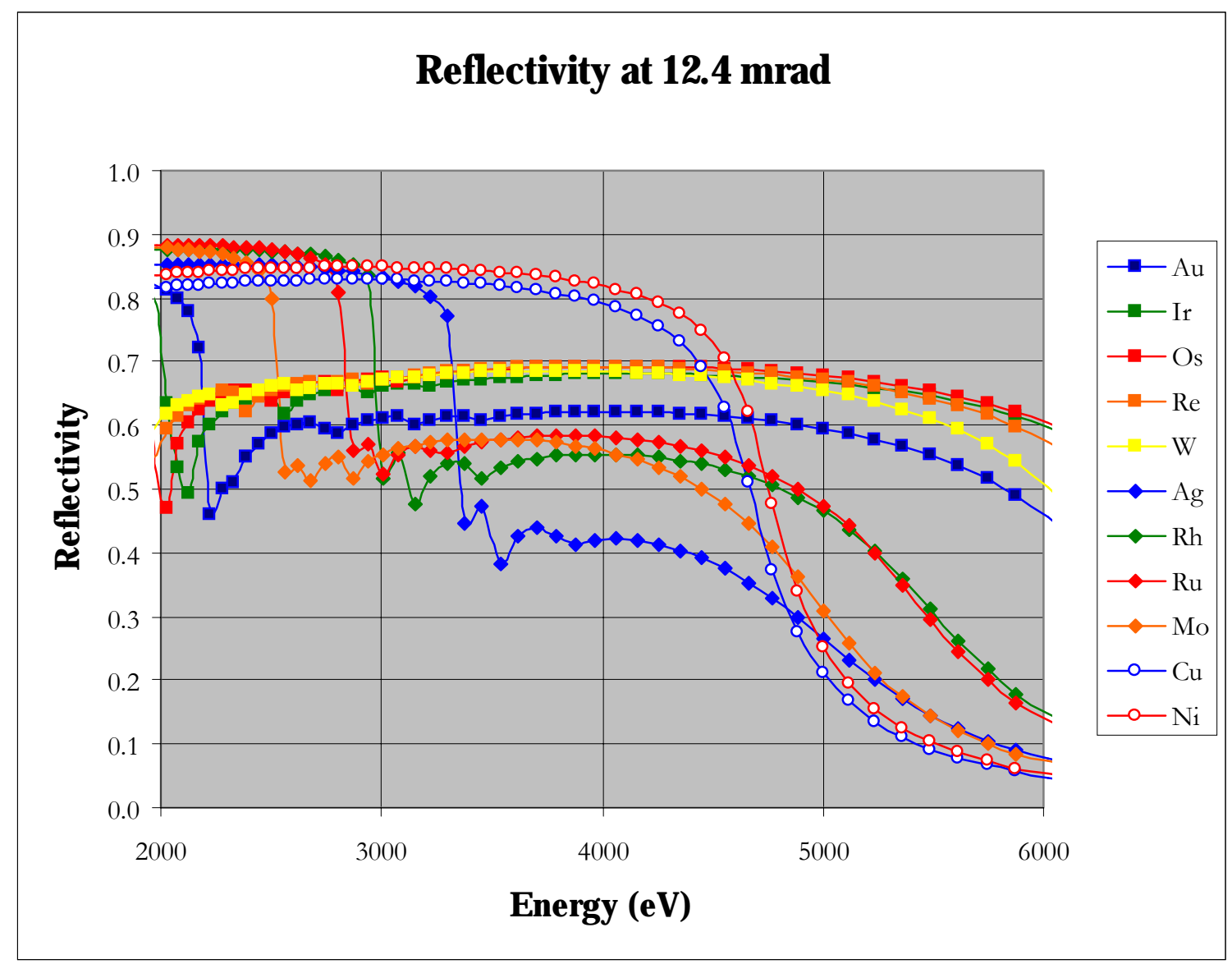

Figure 3.1. The reflectivity of selected refractory and precious metals at a grazing angle of $12.4 \mathrm{mrad}$.

The material of choice for the inner, higher energy, reflective surface is $\mathrm{Ni}$, as described in Chapter 2, and show in Figure 3.1 with hollow red circles. It is immediately obvious that both $\mathrm{Ni}$ and $\mathrm{Cu}$ out perform the row five metals-Ag, $\mathrm{Rh}, \mathrm{Ru}$ and $\mathrm{Mo}-$ despite their lower density. While they out perform both $\mathrm{Ni}$ and $\mathrm{Cu}$ below $2800 \mathrm{eV}$, their reflectivity rolls off very quickly between $2000-3300 \mathrm{eV}$. It could be argued that both $\mathrm{Rh}$ and $\mathrm{Ru}$ out perform $\mathrm{Ni}$ and $\mathrm{Cu}$ above about $4800 \mathrm{eV}$. However the row six metals-Au, Ir, Os, Re and W-out perform even them at higher energies-but fail $\mathrm{Ni}$ and $\mathrm{Cu}$ even worse below $4800 \mathrm{eV}$. Thus, a replacement of Ni by one of the row six metals, perhaps Au or W, is not an ideal choice for extending the high energy performance of the LEXS optic, as it seriously compromises the middle energy range performance..

It was discussed in Chapter 2 how the middle reflective surface, $\Sigma_{2}$, was chosen to be Au to "fill in" the absorption edge of Ni. Figure 3.2 shows the same selected metals at a grazing angle of $25.6 \mathrm{mrad}$, corresponding to the far end of the middle reflective surface. 


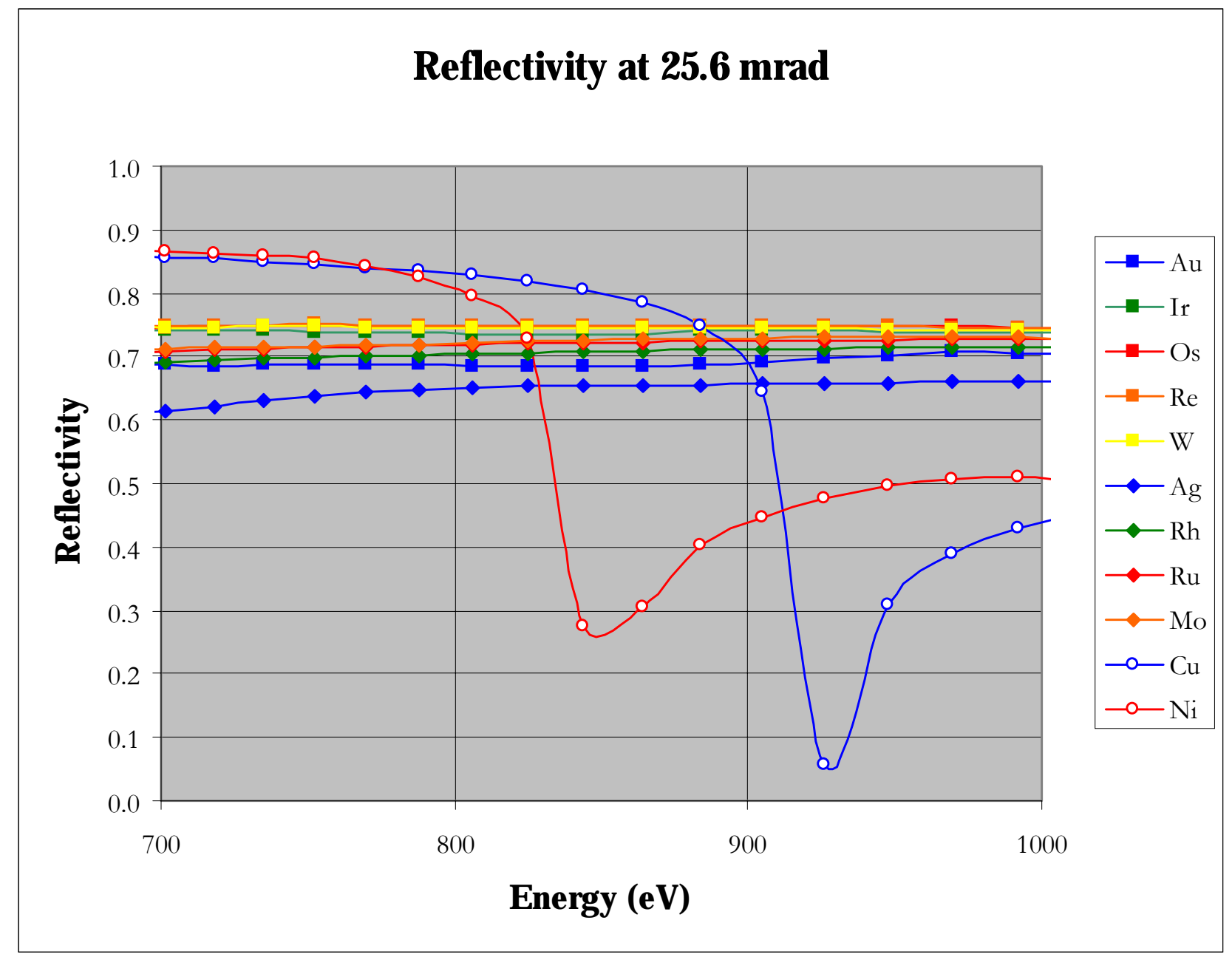

Figure 3.2. The reflectivity of selected refractory and precious metals at a grazing angle of $25.6 \mathrm{mrad}$.

While a choice of any of the other refractory of precious metal other than Ag would lead to a slightly larger reflectivity in the region displayed of $700-1000 \mathrm{eV}$, the region of the Ni Labsorption edge, the differential change would be no more than $8.7 \%$. Since this is a differential increase over a reflectivity of approximately $70 \%$, the increased performance would not immediately warrant the cost of developing technologies for synthesizing nonstrained refractory metal surfaces. As mentioned in Chapter 2, refractory metals, due to their 
hardness, often form high defect density films as they fracture to relieve internal strains. Thus, there is little hope of a substantial performance enhancement by changing the material for surface $\Sigma_{2}$.

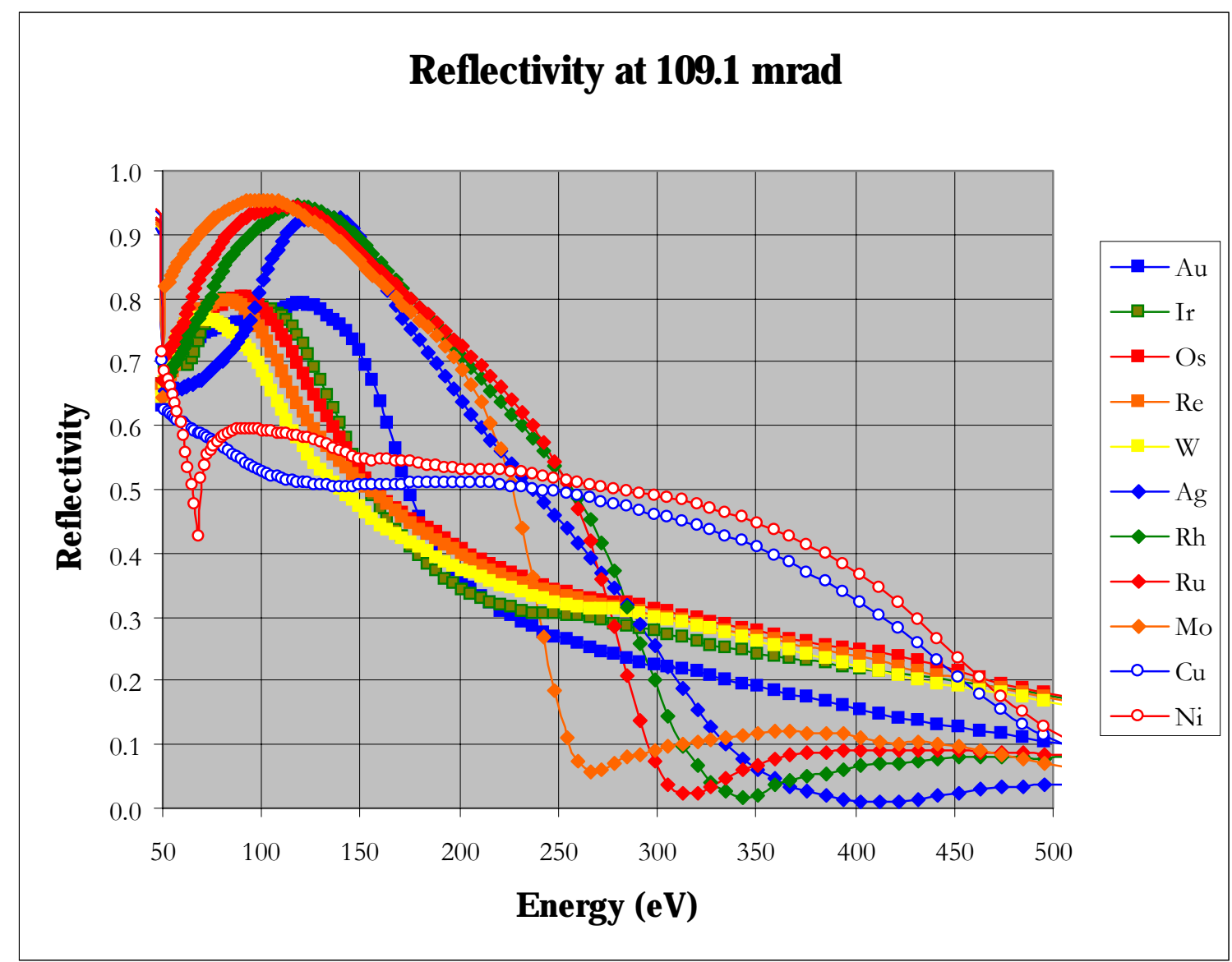

Figure 3.3. The reflectivity of selected refractory and precious metals at a grazing angle of $109.1 \mathrm{mrad}$.

Figure 3.3 shows the reflectivity of these selected metal reflecting surfaces at 109.1 mrad, corresponding to the front end of the outer reflecting surface, $\Sigma_{1}$, the portion key to the low-energy performance. By replacing $\mathrm{Ni}$ with one of the row five metals- $\mathrm{Ag}, \mathrm{Rh}, \mathrm{Ru}$, or Mo-the performance below $250 \mathrm{eV}$ can be greatly enhanced. At $100 \mathrm{eV}$, Mo would increase the reflectivity by approximately $50 \%$, and at $200 \mathrm{eV}$, Ru would increase reflectivity by approximately $36 \%$. These are modifications of potential value in the future prototyping of very low-energy optic. Such an optic would be limited to an energy range of 0-300 eV if an arbitrary reflectivityi cut-off of 0.2 is set for minimum optic utility. Ni extends the same standard for performance to $450 \mathrm{eV}$. At the $\mathrm{Cu} \mathrm{K}$ line $(277 \mathrm{eV})$, Ni out performs the other 
metals. However, a Ru reflective surface would gain approximately $44 \%$ for the B $\mathrm{K}$ line $(183 \mathrm{eV}$ - - but at the cost of less $\mathrm{Cu} \mathrm{K}$ transmission. As the purpose of the NextGen is primarily an enhancement of the mid and high energy performance of the LEXS optic, this finding will be merely noted for future application.

\section{Extending Solid Angle: The NextGen Innovation}

To increase the effective solid angle, which is a product of the accepted solid angle and reflectivity integrated over the reflective surface, either the reflectivity of the optic surface, or the solid angle of acceptance must be increased. The calculation of the effective solid angle of the LEXS optic was discussed in Chapter 2. As discussed above, materials choices alone will not afford a significant extension of the high energy LEXS performance without a compromise of the existing low and mid-energy performance. Thus, any significant LEXS optic performance enhancement must come from an either an increase in total collected solid angle or a reduction of grazing angle without simultaneously reducing the collected solid angle.

A larger optic would demand larger diffractors and subsequently larger proportional counter, a problem which presents cost limitations and detector window limitations. More importantly, a larger collected solid angle must not result in increased grazing angles, as this will result in a reduction of the integrated reflectivities and thus the total effective solid angle. It is impossible to achieve both aims simultaneously by merely increasing the size of the reflecting optics. As indicated in Equation 2.5 in Chapter 2, the larger the acceptance angle of the reflective surface, the larger the collected solid angle, but as shown in Equation 1.13, the larger the acceptance angle, the larger the grazing angle. The innovation of the NextGen design is to exploit two focal points - a near one for solid angle collection, and a distant one for reflection-to reduce grazing angle and thus increase reflectivity without subsequently reducing the collected solid angle as is normally the case. 


\section{NextGen: The Concept}

The NextGen optic consists of both hyperboloidal and paraboloidal sections, both of which are surfaces of revolution. See Figure 3.4. The front portion of the optic is a hyperboloidal section, while the remainder of the optic is a paraboloidal section. The portions of the surfaces representing the optic are indicated with a red broken line in Figure 3.4. The origin of the hyperboloid of revolution is $\mathrm{O}_{\mathrm{H}}$, while the origin of the paraboloid of revolution is $\mathrm{O}_{\mathrm{p}}$, with the axis of revolution being the z-axis. The X-ray source is placed at

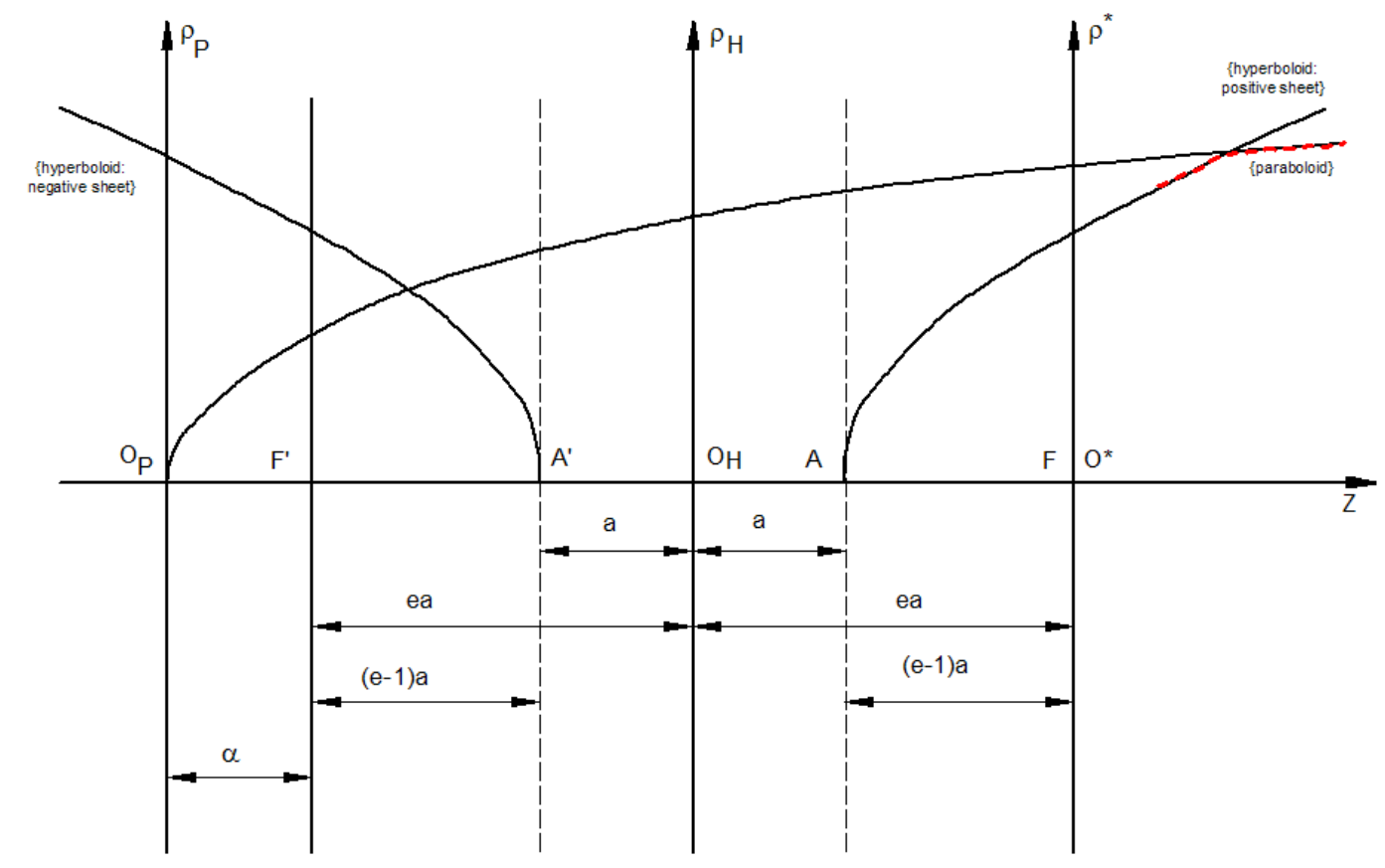

Figure 3.4. Geometry of the NextGen optic. Red broken line indicates surface of actual optic.

the origin, $\mathrm{O}^{*}$, of the positive hyperboloidal sheet. X-rays reflecting from the hyperboloidal surface subsequently reflect from the paraboloidal section as if they were originating from origin $\mathrm{O}_{\mathrm{P}}$, which is distance $\alpha+2 \mathrm{a} \varepsilon$ from $\mathrm{O}^{*}$. This displacement of the effective source of $\mathrm{X}$-rays reduces the maximum grazing angle on the paraboloidal surface without aditionally reducing collected solid angle.

The original LEXS optic is a single reflection optic. Each ray originating from the focus reflects from the paraboloidal surface once before it emerges collimated. The NextGen optic is of the simplest class of multiple reflection optic: each ray undergoes no 
more than two reflections. While it could be argued that multiple reflections would lead to a lower net integrated reflectivity over the interior reflective surface, the NextGen optic outweighs that potential reduction of reflectivity by reducing the grazing angle of each reflection. In the current implementation, the maximum grazing angle, $\gamma_{\max }$, at the front end of the optic - the hyperboloidal end - is variationally optimized to also be the maximum grazing angle at $z_{0}$, the point where the hyperboloidal and paraboloidal surfaces meet. Thus, rays reflecting off the hyperboloidal sheet will have a grazing angle, $\gamma \leq \gamma_{\max }$, with subsequent reflections also having $\gamma \leq \gamma_{\max }$. In addition, rays undergoing one reflection, from the paraboloidal surface alone, will also have $\gamma \leq \gamma_{\max }$. If $r_{\text {min }}=r\left(\gamma_{\max }\right)$, then for all two reflection events (hyperboloidal and paraboloidal reflection), $r \geq\left(r_{\min }\right)^{2}$, and for all one reflection events, $r \geq r_{\text {min }}$.

\section{NextGen: The Theory}

The equation of a hyperboloid of two sheets with the origin shifted to the focus of the positive sheet, $\mathrm{O}^{*}$, is:

$$
\frac{(z+e a)^{2}}{a^{2}}-\frac{\rho^{2}}{\left(e^{2}-1\right) a^{2}}=1 .
$$

$a$ is the major axis of the hyperboloid of revolution and $e$ is the eccentricity of the hyperboloid where

$$
e=\frac{\sqrt{a^{2}+b^{2}}}{a} \text {. }
$$

$z$ is the position along the axis of revolution, and $\rho$ is the radius from the axis of revolution, as with cylindrical coordinates. The equation of a paraboloid with its origin shifted to the focus of the negative sheet of the paraboloid of revolution, $\mathrm{O}_{\mathrm{P}}$, is:

$$
\rho^{2}=4 \alpha(z+\alpha+2 \alpha e)
$$


As in Chapter 1, one obvious constraint is the radius of the largest end of the optic, in this case, the largest radius of the paraboloidal sheet, $w$ :

$$
w^{2}=4 \alpha(h+\alpha+2 e a)
$$

where $h$ is defined as in Chapter 1 , as the distance between the focus of the optic, O*, and the far end of the optic, the end of the paraboloidal section. $\alpha$, the shape parameter of the paraboloidal surface, can then be obtained by taking the positive root of Equation 3.4:

$$
\alpha=-\frac{h}{2}-e a+\frac{1}{2} \sqrt{h^{2}+4 h e a+4 e^{2} a^{2}+w^{2}}
$$

With the paraboloidal surface defined by the maximal radius of the optic, $\alpha$ can then be used, together with the maximum grazing angle, $\gamma_{\max }$, of the front end of the optic, to find $a$ and $e$, and thus define the hyperboloidal surface. The procedure is quite similar to that of Chapter 1. See Figure 1.1. The maximum acceptance angle, $\theta_{\max }$, entering the front hyperboloidal surface of the optic is defined by

$$
\tan \theta_{\max }=\frac{v}{k}
$$

where $v$ and $k$ are defined as in Chapter 1. $v$ is the radius of the front of the optic, and $k$ is the distance between the focus, $\mathrm{O}^{*}$, and the front of the optic.

The slope of the optic at its front edge, at $\theta_{\max }$, is

$$
m=\tan \phi_{\max }=\frac{d \rho}{d z}(z=k, \rho=v)=\frac{\left(e^{2}-1\right)(k+e a)}{v}
$$

Since (See Figure 1.1)

$$
\theta=\phi+\gamma
$$


then

$$
\tan (\theta-\phi)=\frac{\tan \theta-\tan \phi}{1+\tan \theta \tan \phi}=\tan \gamma
$$

With $\gamma_{\max }$ fixed as design constraint, as in Chapter 1 , and with $\theta_{\max }$ and $\phi_{\max }$ defined above, Equation 3.9 can be solved for $a$

$$
a=\frac{v^{2}-\left(e^{2}-1\right) k^{2}-v k e^{2} \tan \gamma_{\max }}{e\left(\left(e^{2}-1\right)\left(v \tan \gamma_{\max }+k\right)\right)}
$$

which still includes the unknown, $e$.

The additional constraint of the radius of the front end of the optic, $v$, can be used to now calculate the eccentricity of the hyperboloidal sheet, $e$. Inputting $z=k$ and $\rho=v$ into Equation 3.1 and solving for the positive root:

$$
a=\frac{-k e+\sqrt{v^{2}+k^{2}}}{\left(e^{2}-1\right)} .
$$

Equating the two solutions for $a$, Equation 3.11 and 3.10, and solving for $e$

$$
e=\frac{\sqrt{v^{2}+k^{2}}}{v \tan \gamma_{\max }+k} .
$$

Substuting $e$ in Equation 3.12 back into Equation 3.11, one obtains a solely in terms of $v, k$ and $\gamma_{\max }$ :

$$
a=\frac{\sqrt{v^{2}+k^{2}}\left(1-\frac{k}{v \tan \gamma_{\max }+k}\right)}{\left(\frac{v^{2}+k^{2}}{\left(v \tan \gamma_{\max }+k\right)^{2}}\right)-1} .
$$


Some internal checks can be performed upon these results. $a>0$, for all $v, k$ and $\gamma_{\max }$, as is expected. $e>1$ as is expected for a hyperboloid of two sheets. From Equation 3.12, the following constraint is immediately evident:

$$
\frac{\sqrt{v^{2}+k^{2}}-k}{v}>\tan \gamma_{\max }>0 .
$$

$a=a\left(v, k, \gamma_{\max }\right)$ and $e=e\left(v, k, \gamma_{\max }\right)$. Since $\alpha=\alpha(a, h, e, w)$, and $e=e\left(v, k, \gamma_{\max }\right)$, then $\alpha=\alpha\left(h, v, k, \gamma_{\max }\right)$. Substituting Equations 3.12 and 3.13 into Equation 3.5 one obtains for $\alpha$ :

$$
\begin{gathered}
\alpha=-\frac{h}{2}-\frac{\sqrt{v^{2}+k^{2}}\left(-\frac{k \sqrt{v^{2}+k^{2}}}{v \tan \gamma_{\max }+k}+\sqrt{v^{2}+k^{2}}\right)}{\left(v \tan \gamma_{\max }+k\right)\left(\frac{v^{2}+k^{2}}{\left(v \tan \gamma_{\max }+k\right)^{2}}-1\right)} \\
+\frac{1}{2} \sqrt{\frac{4 \sqrt{v^{2}+k^{2}}\left(-\frac{k \sqrt{v^{2}+k^{2}}}{v \tan \gamma_{\max }+k}+\sqrt{v^{2}+k^{2}}\right)}{\left(v \tan \gamma_{\max }+k\right)\left(\frac{v^{2}+k^{2}}{\left(v \tan \gamma_{\max }+k\right)^{2}}-1\right)}\left(h+\frac{\sqrt{v^{2}+k^{2}}\left(\sqrt{v^{2}+k^{2}}-\frac{k \sqrt{v^{2}+k^{2}}}{v \tan \gamma_{\max }+k}\right)}{\left(v \tan \gamma_{\max }+k\right)\left(\frac{v^{2}+k^{2}}{\left(v \tan \gamma_{\max }+k\right)^{2}}\right)}\right)}
\end{gathered}
$$

The actual implementation of the NextGen optic requires the point of intersection between the front hyperboloidal surface and the end paraboloidal surface, $z_{0}$. This point is required for the machining of the appropriate mandrels, as well as for any calculation or simulation of the NextGen optic performance. Equating Equations 3.1 and 3.3 and solving for $z$, one obtains $z_{0}$ :

$$
z_{0}=\frac{1}{e-1}\left(2(\alpha+e a)-\left(e^{2}+1\right) a\right)
$$


where it should be remembered, as indicated above, that $\alpha, a$ and $e$ are ultimately functions of $v, k, w$ and $\gamma_{\max }$

\section{NextGen Implementation}

As with the original LEXS paraboloidal optic, the NextGen hyperboloidalparaboloidal optic can be implemented alone, as a stand-alone optic, or as a nested set of coaxial reflecting surfaces. Anticipated prototype implementations of the NextGen optic are: 1) a fourth NextGen optic nested within the existing LEXS optic, 2) a series of nested hyperboloidal-paraboloidal NextGen optics. While the other three LEXS paraboloidal optics could gain some additional effective solid angle, the primary motivation of the NextGen design has been to extend the usable energy range of the LEXS optic. As is shown in Figure 2.7, the total effective solid angle of the existing LEXS optic is less than 10 mster above $2200 \mathrm{eV}$. That is only $25 \%$ of the roughly 40 mster at $1000 \mathrm{eV}$, and about $8 \%$ of the roughly 130 mster at $250 \mathrm{eV}$. This section will describe a solution of the NextGen design problem which utilizes the same paraboloidal reflective surface, $\Sigma_{1}$, as used in the existing LEXS optic. In this implementation, the NextGen hyperboloidal-paraboloidal optics will nest within the outermost LEXS paraboloidal optic, $\Sigma_{1}$, described in Chapters 1 and 2. As it is explained in detail in Chapter 1, the formalism for guaranteeing that nested reflective surfaces do not block each other's collected solid angle will not be revisited here. The procedure is essentially the same for nested paraboloidal surfaces as it is for nested hyperboloidal-paraboloidal surfaces.

\section{NextGen Solution: A Likely Implementation}

Table 3.1 shows one of the most likely implementations of the NextGen tworeflection approach to increasing effective solid angle. As discussed above, the outer optic was chosen to be the existing LEXS paraboloidal reflective surface, $\Sigma_{1}$. Thus, the length of the optic was maintained at approximately $6.5 \mathrm{~cm}$, with $k=1.9888 \mathrm{~cm}$ and $h=8.4888 \mathrm{~cm}$ as in Chapter 1. The maximum grazing angle of $109.09 \mathrm{mrad}$ for $\Sigma_{1}$ was also maintained. Table 3.1 indicates which surfaces are paraboloidal (LEXS) and which are hyperboloidal- 


\begin{tabular}{|c|c|c|c|c|c|c|}
\hline Optic & Type & $\mathrm{k}(\mathrm{cm})$ & $\mathrm{h}(\mathrm{cm})$ & $\mathrm{v}(\mathrm{cm})$ & $\mathrm{w}(\mathrm{cm})$ & $\gamma_{\max }(\mathrm{mrad})$ \\
\hline$\Sigma_{1}$ & LEXS & 1.9888 & 8.4888 & 0.4356 & 0.9000 & 109.09 \\
\hline$\Sigma_{2}$ & NextGen & 1.9888 & 8.4888 & 0.2074 & 0.4356 & 32.11 \\
\hline$\Sigma_{3}$ & NextGen & 1.9888 & 8.4888 & 0.1021 & 0.2074 & 15.89 \\
\hline$\Sigma_{4}$ & NextGen & 1.9888 & 8.4888 & 0.0486 & 0.1021 & 7.54 \\
\hline$\Sigma_{5}$ & NextGen & 1.9888 & 8.4888 & 0.0240 & 0.0486 & 3.72 \\
\hline
\end{tabular}

\begin{tabular}{|l|l|l|l|l|}
\hline Optic & $\mathrm{e}$ & $\mathrm{a}(\mathrm{cm})$ & $\mathrm{b}(\mathrm{cm})$ & $\alpha$ \\
\hline$\Sigma_{1}$ & & & & 0.023850 \\
\hline$\Sigma_{2}$ & 1.00206 & 1.0000 & 0.0643 & 0.004519 \\
\hline$\Sigma_{3}$ & 1.00050 & 1.0000 & 0.0316 & 0.001025 \\
\hline$\Sigma_{4}$ & 1.00011 & 1.0000 & 0.0151 & 0.000248 \\
\hline$\Sigma_{5}$ & 1.00003 & 1.0000 & 0.0074 & 0.000056 \\
\hline
\end{tabular}

Table 3.1. Results for NextGen design implementation.

paraboloidal (NextGen). The outer paraboloidal LEXS optic, $\Sigma_{1}$, was chosen to accept, as in Chapter 1, approximately 150 mster, thus fixing $v_{l}=0.4356 \mathrm{~cm}$.

The parameters in Table 3.1 taken as design constraints are shown in italics. One additional constraint of maintaining $a$ constant was imposed. The reason for this constraint is to fix the relationship between the foci of the paraboloid and hyperboloid, which is at the heart of the NextGen design principal itself. Other models where $a$ is nonconstant are being investigated. For convenience, $a$ was also set to a convenient value of 1.0000. The effect of $a$ on performance is being investigated as well. There is still one free contraint due to the fact that there are two reflective surfaces: their intersection point. Instead of setting $z_{0}$, the intersection point, to a constant value, the maximum grazing angle, $\gamma_{\max }$, was tuned so as to make the grazing angle at $z_{0}$ be $\gamma_{\max }$ as well. The implications of this is that any rays reflecting from the hyperboloidal sheet onto the paraboloid will have a grazing angle, $\gamma \leq$ $\gamma_{\max }$, while also allowing any rays reflecting from just the paraboloid alone to have $\gamma \leq \gamma_{\max }$. 
This optimization was done variationally until $\gamma(\mathrm{z}=\mathrm{k})=\gamma\left(\mathrm{z}=z_{0}\right)=\gamma_{\max }$. The values for $z_{0}$ are in the range of $4.08-4.38 \mathrm{~cm}$, with small variations between reflective surfaces. Due to the constraint that an optic not block the solid angle of the next inner optic, the remaining design parameters were obtainable from those of $\Sigma_{1}$ alone. The procedure closely parallels that outlined in Chapter 1.

\section{Summary of Projected Performance Enhancements: LEXS vs. NextGen}

Table 3.2 summarizes the design parameters of the existing LEXS optic for the sake of comparison. Again, italicized entries are taken as design constraints. Two things are immediately evident. First, the maximum grazing angles for the surfaces $\Sigma_{2}$ and $\Sigma_{3}$ are smaller for the NextGen optic implementation than for the LEXS implementation: 32.11 mrad vs. $52.90 \mathrm{mrad}$, and $15.89 \mathrm{mrad}$ vs. $25.65 \mathrm{mrad}$. That is a reduction of $\gamma_{\max }$ of $39 \%$ for $\Sigma_{2}$ and $38 \%$ for $\Sigma_{3}$

\begin{tabular}{|c|c|c|c|c|c|c|}
\hline Optic & $\mathrm{k}(\mathrm{cm})$ & $\mathrm{h}(\mathrm{cm})$ & $\mathrm{v}(\mathrm{cm})$ & $\mathrm{w}(\mathrm{cm})$ & $\gamma_{\max }(\mathrm{mrad})$ & $\alpha$ \\
\hline$\Sigma_{1}$ & 1.9888 & 8.4888 & 0.4356 & 0.9000 & 109.09 & 0.023850 \\
\hline$\Sigma_{2}$ & 1.9888 & 8.4888 & 0.2104 & 0.4356 & 52.90 & 0.005550 \\
\hline$\Sigma_{3}$ & 1.9888 & 8.4888 & 0.1015 & 0.2104 & 25.65 & 0.001295 \\
\hline
\end{tabular}

Table 3.2. Results for LEXS design implementation.

Thus the proposed increase in reflectivity should be achieved on the basis of this significant reduction of grazing. Figure 3.5 below shows the reflectivity of $\mathrm{Ni}$ and $\mathrm{Au}$, the reflective metals used in the LEXS optic, at $1500 \mathrm{eV}$ over a range of grazing angle of 0.0$60.0 \mathrm{mrad}$. Data was calculated using SF, using bulk densities and $1000 \mu \mathrm{m}$ film thickness. At the LEXS maximum grazing angle for $\Sigma_{2}$ of $52.9 \mathrm{mrad}$, one anticipates a reflectivity of $3.9 \%$ for $\mathrm{Ni}$ and $7.9 \%$ for $\mathrm{Au}$. If the NextGen prototype works, and $\gamma_{\max }$ is reduced to $32.11 \mathrm{mrad}$, one can then anticipate a reflectivity of $44.5 \%$ for $\mathrm{Ni}$ and $56.1 \%$ for Au. This is a $11 \mathrm{X}$ increase for $\mathrm{Ni}$ and $7 \mathrm{X}$ increase for $\mathrm{Au}$. 


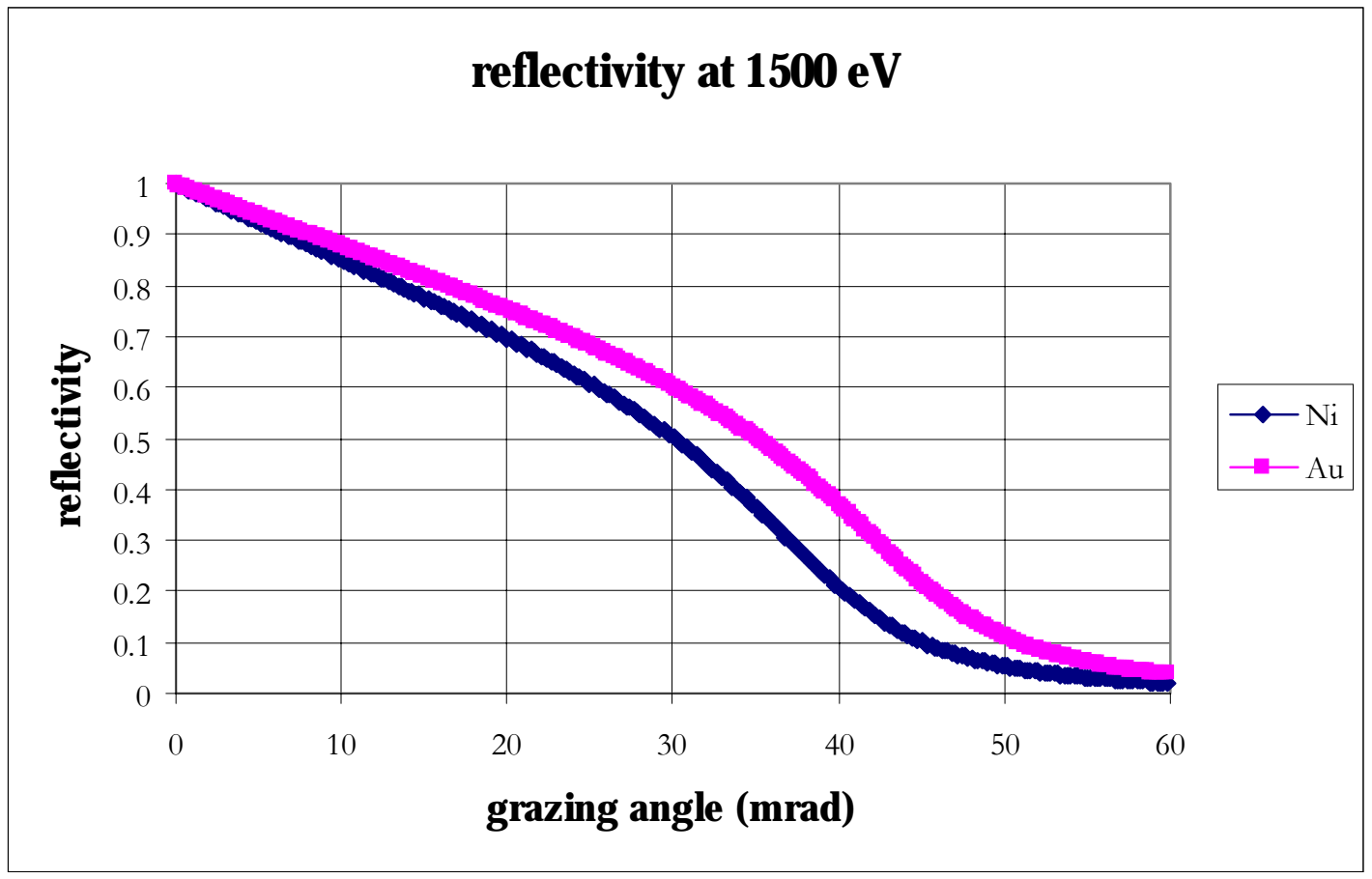

Figure 3.5. Reflectivity of $\mathrm{Ni}$ and $\mathrm{Au}$ at $1500 \mathrm{eV}$.

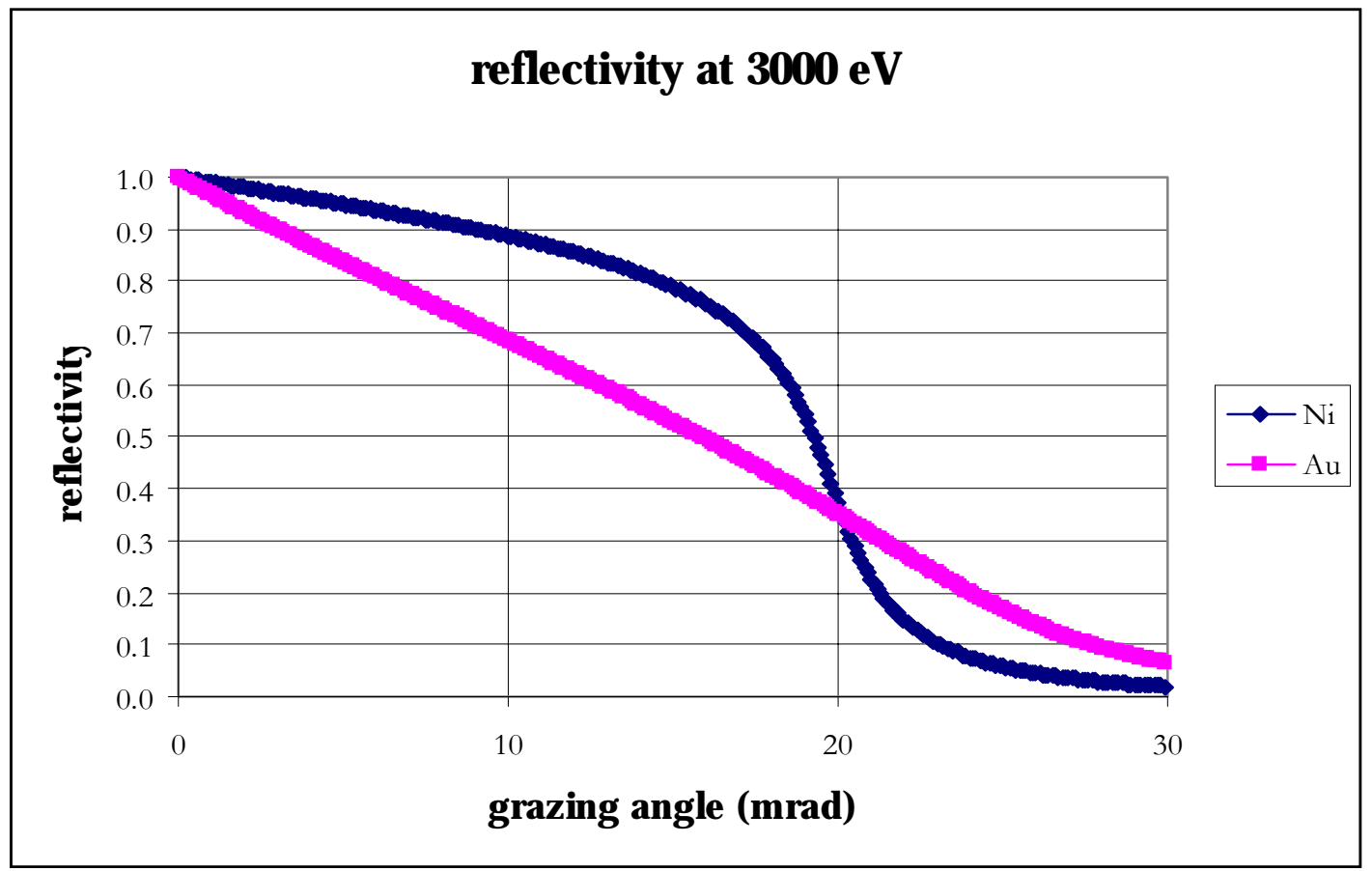

Figure 3.6. Reflectivity of $\mathrm{Ni}$ and $\mathrm{Au}$ at $3000 \mathrm{eV}$. 
Figure 3.6 shows the reflectivity of $\mathrm{Ni}$ and $\mathrm{Au}$ at $3000 \mathrm{eV}$. At the LEXS maximum grazing angle for $\Sigma_{3}$ of $25.65 \mathrm{mrad}$, one anticipates a reflectivity of $4.9 \%$ for $\mathrm{Ni}$ and $14.8 \%$ for $\mathrm{Au}$. If the NextGen prototype works, and $\gamma_{\max }$ is reduced to $15.89 \mathrm{mrad}$, one can then anticipate a reflectivity of $75.9 \%$ for $\mathrm{Ni}$ and $49.9 \%$ for $\mathrm{Au}$. This is a $15 \mathrm{X}$ increase for $\mathrm{Ni}$ and 3.4X increase for Au.

These are non-trivial enhancements of reflectivity. While the above figures are for one grazing angle, $\gamma_{\max }$, it should be noted that gmax is the maximum grazing angle for rays refleting from both the hyperboloidal and paraboloidal surfaces since $\gamma_{\max }$ was variationally optimixed with $\gamma(\mathrm{z}=\mathrm{k})=\gamma\left(\mathrm{z}=z_{0}\right)=\gamma_{\max }$ as a constraint. Thus, all rays will have a grazing angle less than $\gamma_{\max }$, and thus reflectivities larger than those stated above. The integrated reflectivities over the interior surfaces of the combined hyperboloidal-paraboloidal surface will thus be larger than those stated at $\gamma_{\max }$ as above.

The second observation is that this decrease of maximum grazing angle is achieved without the cost of reduced total collected solid angle. Table 3.3 shows the total reflected solid angle-i.e. total collected solid angle minus the solid angle of rays passing through the optic without reflection-for the LEXS and NextGen optics. It should be recalled that the

\begin{tabular}{|c|c|c|}
\hline Optic & $\Omega_{\mathrm{r}}$ (mster) LEXS & $\Omega_{\mathrm{r}}$ (mster) NextGen \\
\hline$\Sigma_{1}$ & 113.59 & 113.59 \\
\hline$\Sigma_{2}$ & 26.57 & 25.62 \\
\hline$\Sigma_{3}$ & 6.34 & 6.38 \\
\hline$\Sigma_{4}$ & & 1.42 \\
\hline$\Sigma_{5}$ & & 0.35 \\
\hline
\end{tabular}

Table 3.3. Total reflected solid angle for LEXS and NextGen optic implementations.

NextGen uses the same outer optic, $\Sigma_{1}$, as the LEXS implementation. As was desired, there is very little change between the LEXS and NextGen total reflected solid angles.

Conclusion 
From the present work it is evident that the two reflection hyperboloidalparaboloidal optic can reduce the maxinum grazing angle, $\gamma_{\max }$, by "virtually" shifting the focus of the collimating surface - the paraboloid-away from the focus of the collecting surface-the hypberboloid. A direct comparison of $\gamma_{\max }$ between LEXS and NextGen implementations having the same optic length, the same source-optic distance, and the same outer low-energy paraboloidal reflector, shows that the maximum grazing angle can indeed be reduced without trading off solid angle.

Lochner thus comes to similar conclusions as did David OHara when OHara used his simple spreadsheet model. Lochner was able to derive a set of nested reflectors very similar to those derived previously by OHara with a major difference. Lochner's reflectors all have the same $\mathrm{x}$-ray source to entrance aperture distance. This is a major factor in making the final optic easier to assemble and thus far more practical. Lochner was not able to derive the throughput for this optic due to the difficulty of convolving two integrals but his solution is so close to that of OHara's previous calculation of effective solid angle, that we feel confident in using this calculation.

\section{Task 2. $\quad$ Fabricate Interim Optic}

The first problem we had to overcome with fabrication of the interim optic was that we found our existing optics were significantly rougher than we expected. This was found when we were testing an optic for $95 \mathrm{eV}$ and it didn't produce a sharp focal point. We began testing our optics on an AFM at Florida State University and found that our most recent optics that we thought were smooth actually had a roughness of about 30 angstroms rms. By doing many AFM scans of many replicas of the mandrels polished in different ways, we were able to find a technique that seems to result in smooth surfaces. An AFM scan of one of these surfaces is shown in fig. 2. and Table 1. gives some results from this effort. Basically, we determined that very fine (.25 micron) diamond slurry gives a smoother surface than polishing with .05 micron $\mathrm{Al}_{2} \mathrm{O}_{3}$, but, we are not sure why. We also found that polishing often leaves deep scratches oriented in the direction 


\begin{tabular}{|c|c|c|c|c|c|c|c|c|}
\hline Scale & $\begin{array}{l}\text { Small } \\
\text { Nasa } \\
.25 \\
\text { um } \\
\text { Dia } 1 \\
\text { hr }\end{array}$ & $\begin{array}{l}\text { Lrg } \\
\text { NASA } \\
.25 \text { um } \\
\text { dia } 1.75 \\
\text { hr } \# 4\end{array}$ & $\begin{array}{l}\text { Lrg } \\
\text { nasa } \\
\# 6 \\
.5 \mathrm{hr} \mathrm{w} \\
.25 \mathrm{um} \\
1 \mathrm{hr} \text { oil } \\
1.5 \mathrm{hr} \\
1 / 8 \mathrm{um} \\
1 \mathrm{hr} \text { oil }\end{array}$ & $\begin{array}{l}\text { EDS } \\
\text { mandrel } \\
.5 \mathrm{hr} \\
\text { with } \\
.25 \mathrm{um} \\
\text { diamond }\end{array}$ & $\begin{array}{l}\text { Lrg } \\
\text { LEXS } \\
\text { Rot, . } 1 \\
\text { A12O3 } \\
\text { For } 1 \\
\text { hr, .05 } \\
.5 \mathrm{hr}\end{array}$ & $\begin{array}{l}\text { Mid } \\
\text { LEXS, } \\
400 \mathrm{~A} \\
\mathrm{Au}, 1 \\
\mathrm{hr} .05 \\
\mathrm{~A} 12 \mathrm{O} 3\end{array}$ & $\begin{array}{l}\text { Lrg } \\
\text { LEXS } \\
\text { No rot. } \\
\# 7 \\
1 \mathrm{hr} \\
.25 \mathrm{um} \\
1 \mathrm{hr} \text { oil }\end{array}$ & $\begin{array}{l}\text { Small } \\
\text { NASA } \\
.25 \mathrm{um} \\
\text { dia, then } \\
.1 \mathrm{um} \\
\mathrm{Al} 2 \mathrm{O} 3 \\
\text { for } 1 \mathrm{hr}\end{array}$ \\
\hline 1 & 7 Ang. & 23,42 & 6,10 & 16,21 & 14,25 & & 24 & \\
\hline 3 & 9 & & 12 & & & & 30 & \\
\hline 5 & 14 & 32 & 16,17 & $57^{*}$ & 53,57 & 23 & 31,39 & 44,76 \\
\hline 10 & 67 & 30 & 20 & & & 24 & 33,40 & 43,80 \\
\hline 20 & & 29 & 32 & & 70,45 & 55 & 41 & \\
\hline & & & & & & & & \\
\hline & & & & & & & & \\
\hline
\end{tabular}

- had severe contamination

Table 1. AFM roughness results. Samples were polished in various ways as listed in the top row. We looked at roughness at various scale lengths. In most cases, these were replicas from polished mandrels since the mandrel would not fit under the AFM.

of polishing. These scratches are much larger than the micro-scratches that result in polishing. We can minimize them by polishing at 90 degrees to the original direction for a while and then without changing polishing pads, doing the final polishing in the longitudinal direction. 


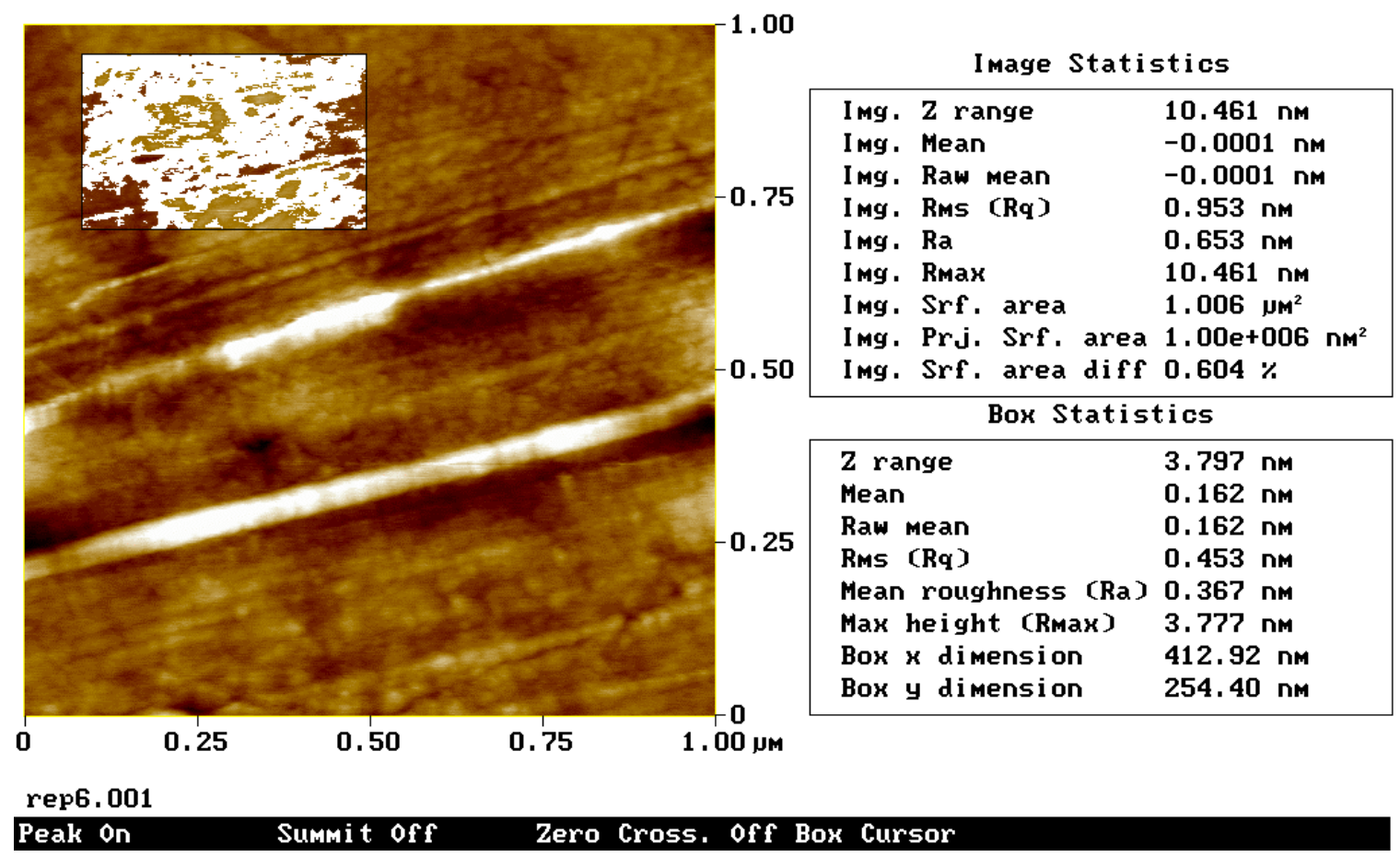

Fig. 2. AFM scan of a replica of a polished mandrel. The diamond turning marks were circumferential and have been removed. What remains are longitudinal polishing grooves. This scan clearly shows large scratches from a previous coarser grade of diamond abrasive showing the need for longer polishing time. However, even with these deep scratches, the overall surface roughness is only $.95 \mathrm{~nm}$ and in an area that does not include these deep scratches, it is less than $.5 \mathrm{~nm}$. Note the remaining scratches produced by the finest grade of diamond leave scratches smaller than the topography from the structure of the electroformed nickel. 


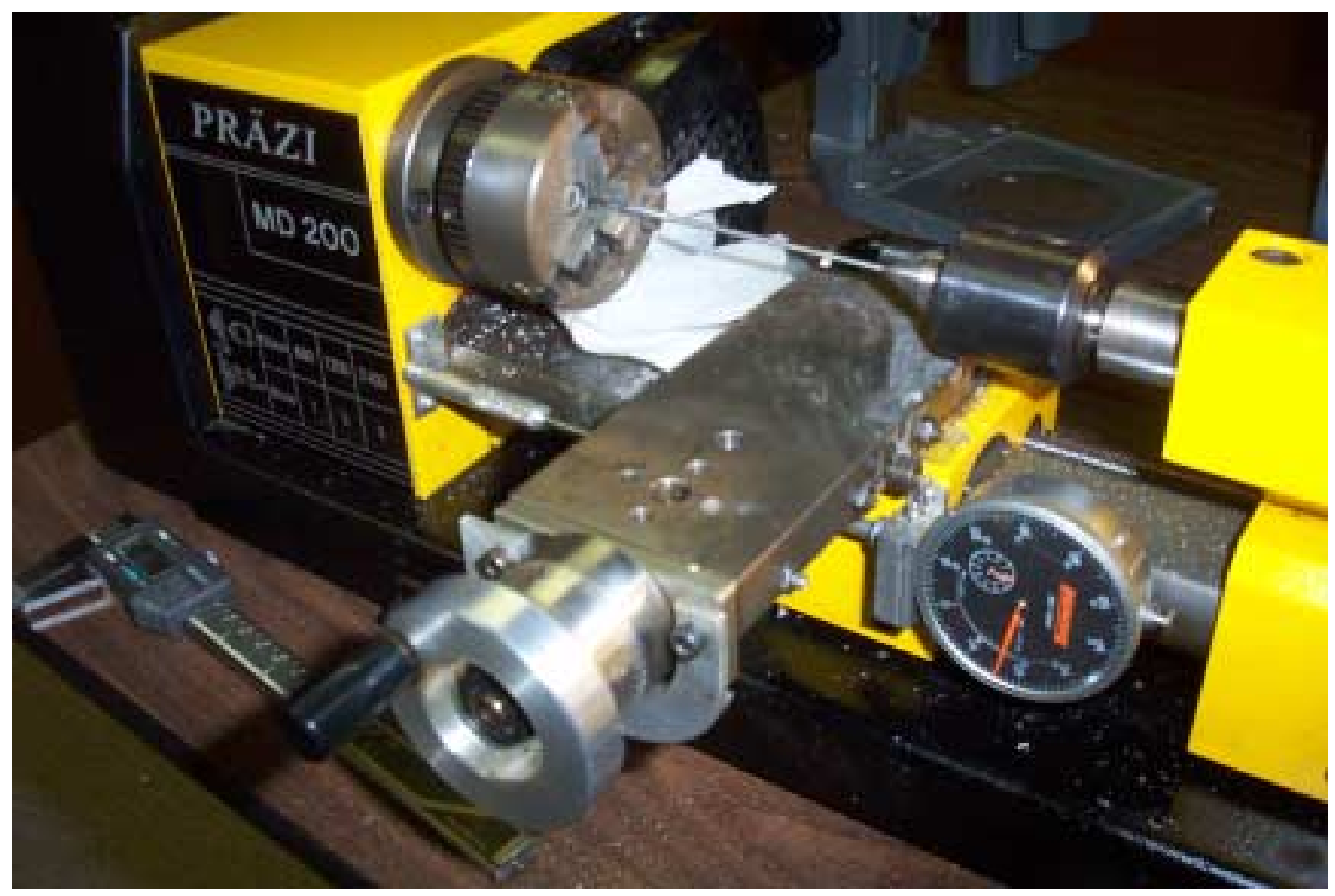

Fig. 3. Fabrication of the mandrel for the Interim Optic. The dial indicator on the Prazi lathe table has been removed so that the mandrel can be seen. The mandrel that is held between the chuck and the "Live Center" was shaped by hand using strips of \#1500 grit carborundum paper.

Our first attempt at making the very small interim optic was a complete failure. We easily shaped the aluminum mandrel and had it coated with electroless nickel. It was easily polished and it looked as if it would be successful but the electroform refused to separate from the mandrel. We never did get it to separate and surmise that this was due to incomplete passivation with the $\mathrm{K}_{2} \mathrm{CrO}_{3}$ solution.

Our next attempt separated very easily with only 10 seconds more in the passivation solution and in fact, it nearly shot off the mandrel. The photo of fig.3. shows the mandrel being polished. We anticipated difficulty in assembling this optic with the previous 3 LEXS reflectors because it is the innermost and the "spokes" that hold it have very tiny notches to catch its edges. In reality, it seemed no more difficult 
than our previous 3 reflector nested optics, an experience that bodes well for the Phase II optics.

\section{Task 3. Testing Interim Optic}

During Phase I, we had planned to test a new diffractor made from HOPG (Highly Oriented Pyrolytic Graphite) with the interim optic. However, since the HOPG arrived early in the effort, we began testing it without the interim optic using only the existing LEXS optics. We didn't expect very good results since the reflectivity of the existing reflectors is low for X-ray energies higher than $2000 \mathrm{eV}$ but we tested anyway. We were surprised to get very good count rates for energies between $2300 \mathrm{eV}$ and $6400 \mathrm{eV}$ (Mo L line to Fe K line). At the Mo line, we got 270 counts/sec/nano-amp (cps/na) with a P/B of 15.8 and a resolution of $49 \mathrm{eV}$. At the $\mathrm{Ti} \mathrm{K}$ line $(4500 \mathrm{eV})$, we got $900 \mathrm{cps} / \mathrm{na}$ with $\mathrm{P} / \mathrm{B}$ of 50 and resolution of $200 \mathrm{eV}$. At the Fe K line, we got about $1000 \mathrm{cps} /$ na with a resolution of $379 \mathrm{eV}$. These count rates are higher than expected but the resolution is worse than expected based on the reported .4 degree wide rocking curve of the graphite. OHara thought this was due to the poor collimation of the x-rays leaving the existing LEXS optic but Lochner suggested that we test the diffractor using the Siemens diffractometer at FSU. Since the LEXS would be unavailable till we were done moving, we decided to do the diffractometer testing. We were surprised to find that initial testing indicated that this piece of graphite has a rocking curve of nearly 1.6 degrees FWHM that corresponds well with the measurements taken with LEXS that correspond to a width of 1.3-1.75 degrees. However, we discussed this with GE Advanced Ceramics who made the graphite and modified the testing procedure and obtained the diffractometer scan in fig.4. indicating a rocking curve of .362 degree FWHM which is better than that specified by the manufacturer.

I directly quote Lochners report of this measurement:

"Using a piece of (012) oriented saphire as a standard (single crystal), I measured the width of the (012) peak and the width of the rocking curve about the (012) peak for the smallest sets of appropriate apertures. Since the correlation length of the saphire can be considered to be infinite, that provides a measure of the broadening inherent in the instrument. I get a peak width of 0.022 deg for the saphire (012) peak and a rocking curve that is 0.024 deg in FWHM.

The width of the (00X) (which might be the (002) or (003) peak of the graphite depending upon the phase) graphite peak is 0.065 degrees, and the rocking curve about this has a FWHM of $0.362 \mathrm{deg}$.

The standard and the diffractor were measured using nonmonochromated $\mathrm{Cu} \mathrm{Ka}$ radiation. The divergence apertures (defining with width of the beam) and the receiving aperture (defining the width of the diffracted beam accepted) were $1.0 \& 0.018 \& 0.1 \& 0.018$ degrees for the peak width and the rocking 
curve measurements. The diffracted beam was monochromated using a graphite monochromator.

What does this mean?

In general terms, the peak width in a diffractometer corresponds to the correlation length (length scale of long range order) convolved with the instrumental broadening which is a function of apertures and diffractometer geometry. The peak widths can be used to estimate the correlation length in the material, which is, in this technique, being probed normal to the diffractor surface. It's a fairly straight forward formalism--

Size $=(K *$ lambda $) /($ width $* \cos ($ theta $))$

Lambda is the wavelength, $K$ is a "structure factor"-- usually $\sim 0.9$, and the width and theta are obvious. Theta is typically corrected for the instrument broadening using subtraction in quadratures

RealWidth $=$ Sqrt $\left(\right.$ measured witdh $^{\wedge} 2-$ width of standard with infinite correationlength $\left.{ }^{\wedge} 2\right)$.

The correlation length isn't as much of an issue as the width of the rocking curve in this case. The very large rocking curve width is suggesting there is a board distribution of crystallites satisfying the Bragg condition. (By definition with a rocking curve the Bragg angle is set and the sample is rocked). A broad rocking curve will reflect broad peaks when used as a monochromator as in WDS. I have no sense why the rocking curve should be so broad, and would like to see the vendor's data. The surface does seem quite "torn up"."

Further discussion of these measurements:

"I did several rocking curves (theta-scans) and theta-2theta coupled scans. DOGA2 is the best looking rocking curve. The structure at the top of it is real, not due to detector saturation. Fitting the peak to two pseudo-Voigt peaks (combos of Lorentzians and Gaussians) with positions fixed at the maxima, gives a peak width for the rocking curve of 0.34 degrees. Fitting it to one gives a FWHM of .59 degrees.

Looking at DOGA6 which has a lot of structure in it... if all of those peaks are fit to pseudo-Voigts with positions fixed at the maxima, one gets pretty much the same FWHM's as found from DOGA2-- they range from 33 to .45 degrees." 

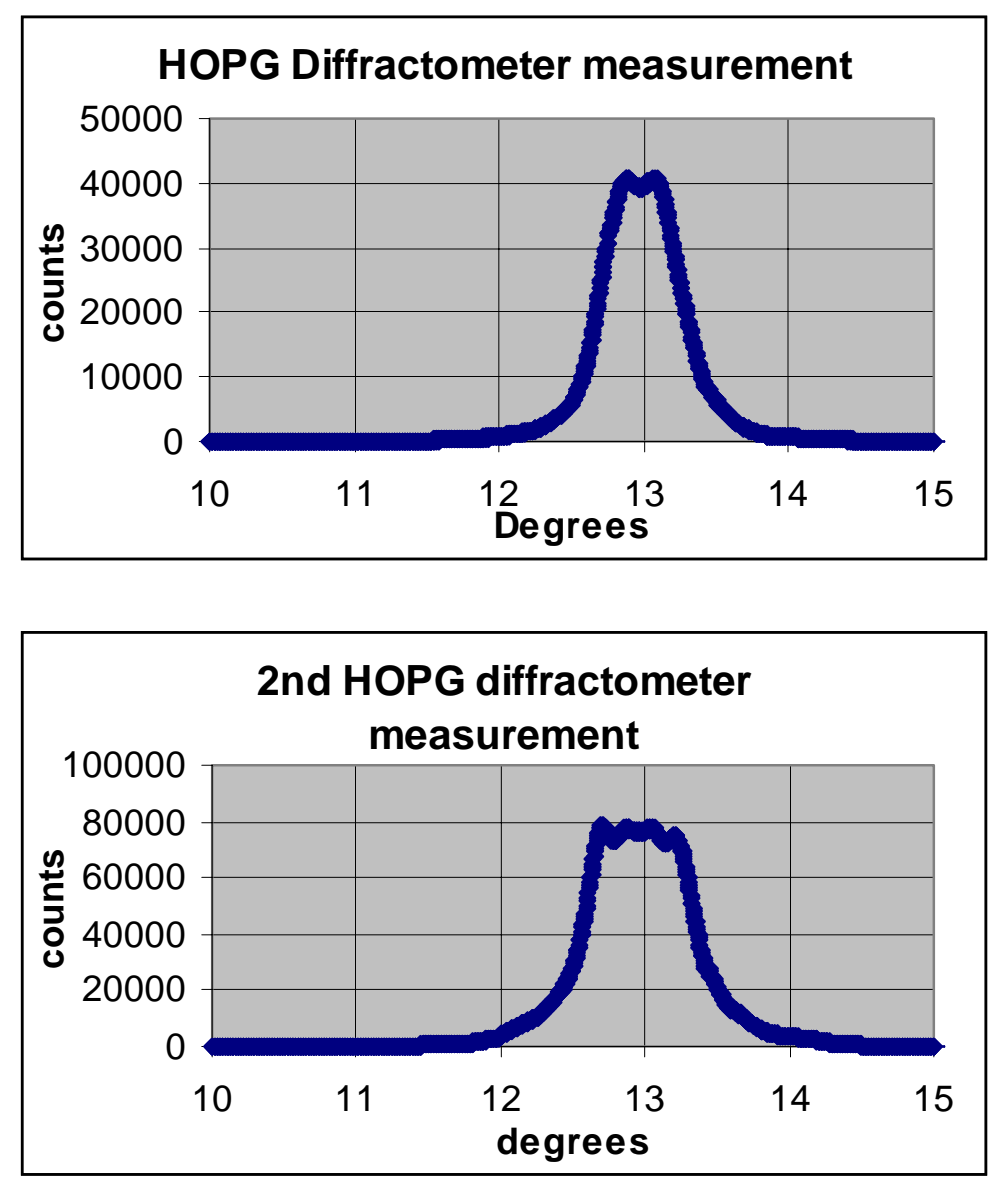

Fig. 4. Two diffractometer scans of the Highly Oriented Pyrolytic Graphite (HOPG) diffractor.

We can think of only two things that might produce a broadening of the resolution to nearly 1.3-1.75 degrees as indicated by our spectrometer data; roughness of the optics that causes scatter of x-rays and the inner cone of x-rays that pass through the collimator without making any reflections. Roughness of the optics can be improved as indicated by the above AFM data and the uncollimated x-rays can be blocked by means of an aperture stop. If we can truly get the .362 degree FWHM, our resolution would be sufficient for many applications including separation of the area around the $\mathrm{Pb}-\mathrm{S}-\mathrm{Mo}$ overlap.

\begin{tabular}{|c|c|c|c|c|}
\hline & $2400 \mathrm{eV}$ & $3500 \mathrm{eV}$ & $4500 \mathrm{eV}$ & 5500 \\
\hline 1.5 degrees & 52 & 145 & 263 & 405 \\
\hline .6 & 21 & 59 & 104 & 162 \\
\hline .4 & 13 & 39 & 70 & 108 \\
\hline
\end{tabular}

Table 2. Top row is x-ray energy; leftmost column is width of rocking curve, so the table entries are diffractor resolution.

From Table 2., it can be seen that if we really can get a graphite diffractor with a .4 degree rocking curve width, then we will be able to separate the $\mathrm{S} / \mathrm{Pb}(36 \mathrm{eV})$ overlap, 
the $\mathrm{Mo} / \mathrm{Pb}(49 \mathrm{eV})$ overlap, and barely separate the $\mathrm{S} / \mathrm{Mo}(13 \mathrm{eV})$ overlap. This diffractor will also be able to separate the Ti/Ba overlap (overlap is $43 \mathrm{eV}$ ) and will be able to get better resolution than any EDS system for the transition elements. If we can only get a type with .6-degree resolution, we will still be able to separate the $\mathrm{S} / \mathrm{Pb}$ overlap and the $\mathrm{Mo} / \mathrm{Pb}$ overlap. A graphite diffractor with .4 degree rocking curve width, used in a fast scanning WDS that has high count rates for most energies could be used to replace EDS entirely

Although the resolution of the graphite diffractor was fairly poor, the high-count rates and good P/B still make it useful for some applications. For example, we have sold a LEXS system to a national lab where they will be measuring impurities in a background of $\mathrm{Pu}$ and they need to be able to see the $\mathrm{Pu}(\mathrm{M})$ line at $3400 \mathrm{eV}$ so that they can use it to align the spectrometer. The graphite diffractor will enable them to easily see this line. We have already done measurements on the $M$ lines of both Thorium and Uranium giving reasonable count rates. Because the WDS using the graphite diffractor is only seeing a narrow spectral region, the SEM can be used at very high beam current to enable a user to collect higher count rates than any EDS system. Using the graphite diffractor enables the LEXS spectrometer to cover the entire Periodic Table with EDS-like resolution where there are few overlaps and using the other diffractors it has very high resolution at lower energies where there are many overlaps.

During the time we were supposed to test this interim optic, Parallax moved to a new facility so our electron microscopes had to be moved. Unfortunately, this caused both our JEOL T300 and JEOL 35 SEMs to fail and even as this is being written, we have not been able to get anybody to service them. In mid-July, 2003, Edax requested that Parallax look into options to improve LEXS performance around the lead-bismuth, molybendum, sulfur overlap for a customer. Our previous results with the graphite diffractor seemed so promising that we reasoned that if the interim optic worked as expected, that it would result in an immediate sale. With this expectation, we sent the interim optic and graphite diffractor to Edax for testing since we were not able to test with our SEMs not working. Edax promptly made a test fixture to hold the graphite diffractor but before they could test, they had to modify their LEXS spectrometer for another purpose and it had to be dismantled and sent to a machine shop. So, as of $8 / 25 / 03$, the interim optic has not been tested. As of $9 / 12 / 03$, Parallax has had their microscopes serviced so they work and we expect to be able to resume testing very soon.

\section{Task 4. Produce Preliminary Design for Full Spectrometer.}

In the Phase I proposal, we discussed the use of six diffractors where the detector is moved in the same way as the current LEXS spectrometer, i.e. with a cam driven by the same axis that drives the diffractor turret. We had thought that using such a cam would be fairly easy and that we would be able to drive it fast enough through its entire range to enable the spectrometer to scan very rapidly. However, our experience with the existing LEXS spectrometer with 5 diffractors is that the limiting factor on the speed of the spectrometer is movement of the detector driven by the cam. Whenever the cam is going 
past one of its 10 vertices, it must slow down or it may stress the detector mount. A sixsided diffractor turret would be even worse. Furthermore, as the detector motion rapidly starts and stops, the detector oscillates because the pin on the detector arm that rides in the cam groove has sufficient play to allow easy movement but this also allows the oscillation. Extreme speed makes the oscillation so severe that we are afraid that we will begin to lose counts. Because of these concerns, we have limited the speed of the LEXS to less than we had originally anticipated.

To solve this problem, we have decided to de-couple the motion of the detector and diffractor turret by driving the detector with its own motor. On first consideration this may seem to be a complication to a fairly simple existing system with only a single motor but in fact the cam is fairly complex and a six-sided cam would be even worse. With a six sided cam, if we want to move to a diffractor 180 degrees away on the turret, the detector must move back and forth 3 times reversing its motion six times in order to reach the desired diffractor. With a decoupled system, the detector would simply go to the desired starting position and stay while the diffractor turret rapidly moved to the desired diffractor. Because the detector is not free to oscillate as with the existing cam, we can drive the diffractor turret as rapidly as possible giving very fast scan speeds.

The utility of the fast scan has already become apparent during demonstrations of the existing LEXS which can only do a fast scan over one diffractor before it must slow down to move to the next one. Users rapidly become accustomed to the fast scan and begin using it to quickly identify major elements before scanning for trace elements in the major element matrix. In a side-by-side test with a conventional WDS, users are surprised that the conventional WDS is unable to do a fast repetitive additive scan as the LEXS can do. Our conclusion from this is that most users will desire new fast scanning modes and that they can easily transition from EDS to a fast scanning WDS.

When David OHara originally designed the LEXS spectrometer, it was intended to only operate below $1 \mathrm{KeV}$ so only had 4 diffractors. Because the diffractors rotate about an axis that is not on the face of the diffractors, when they rotate, they also translate causing the beam to partially miss the diffractor at either very high or very low Bragg angles. Because the analysis of this situation was difficult, OHara designed the LEXS simply by making cutouts of the incident beam and square turret to determine the optimum placement of the incident beam on the diffractor. When Parallax originally decided to expand the LEXS energy range from $1000 \mathrm{eV}$ to $2400 \mathrm{eV}$, this design constraint was forgotten and we simply replaced the old square turret with a 5-sided diffractor turret. On this 5-sided turret, we got lucky in that it worked very well over its entire range of Bragg angles. When we proposed a six sided diffractor turret in Phase I, we had completely forgotten the original design constraint. Fortunately, Dr. Eric Lochner decided to model the x-ray path through the LEXS and found that the five-sided version is the largest number of sides before we would begin to lose significant numbers of x-rays unless we increase the length of the diffractors. Fig. 6 illustrates this showing that for Bragg angles below 20 degrees, that x-rays reflecting from the outermost collimating paraboloid begin to miss the diffractor. This is only a problem for energies from about $100 \mathrm{eV}$ up to $1000 \mathrm{eV}$ and is more of a problem for energies above $500 \mathrm{eV}$ because these energies reflect primarily from the outer diameter of the outermost reflector of the collimator. For a six-sided turret, the situation is even more severe as shown in Fig.7. If we want to keep the mechanically simple rotation about the turret axis instead of 
rotating about the diffractor face, we need to increase the diffractor length by $2 \mathrm{~mm}$ so that all the parallel x-rays diffract from it. Even with this increased length, we lose x-rays for Bragg angles below 20 degrees (for energies below $1000 \mathrm{eV}$ only). The solution to this problem is probably to replace the existing $2 \mathrm{~d}=60 \mathrm{~W} / \mathrm{Si}$ multilayer with a $2 \mathrm{~d}=45$ $\mathrm{W} / \mathrm{Si}$ multilayer that Osmic already sells. This would make all of the x-rays between 500 and $1000 \mathrm{eV}$ have Bragg angles greater than 16 degrees. The only elements that would suffer from loss of counts would be $\mathrm{Ni}$ and $\mathrm{Cu}$ (L lines) but these have such high-count rates now that it isn't expected to be a problem.

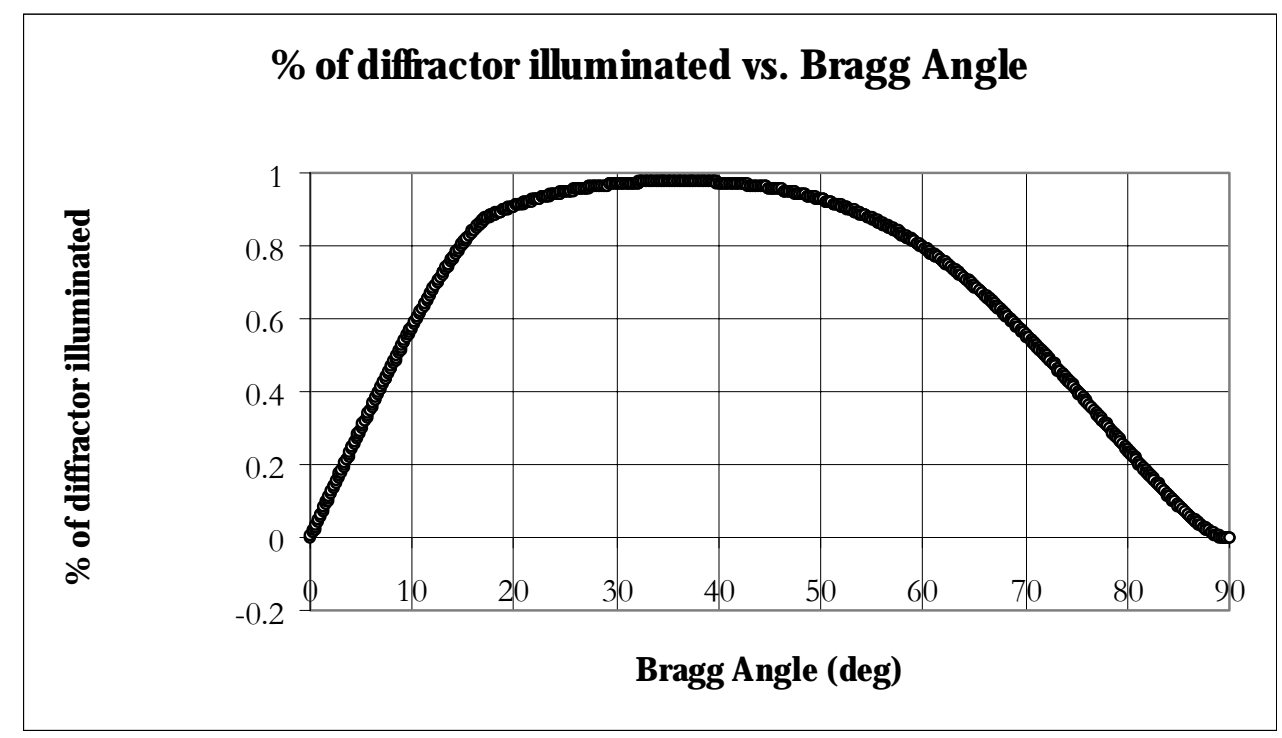

Fig. 6. Fraction of X-rays that hit the diffractor for a five sided turret.

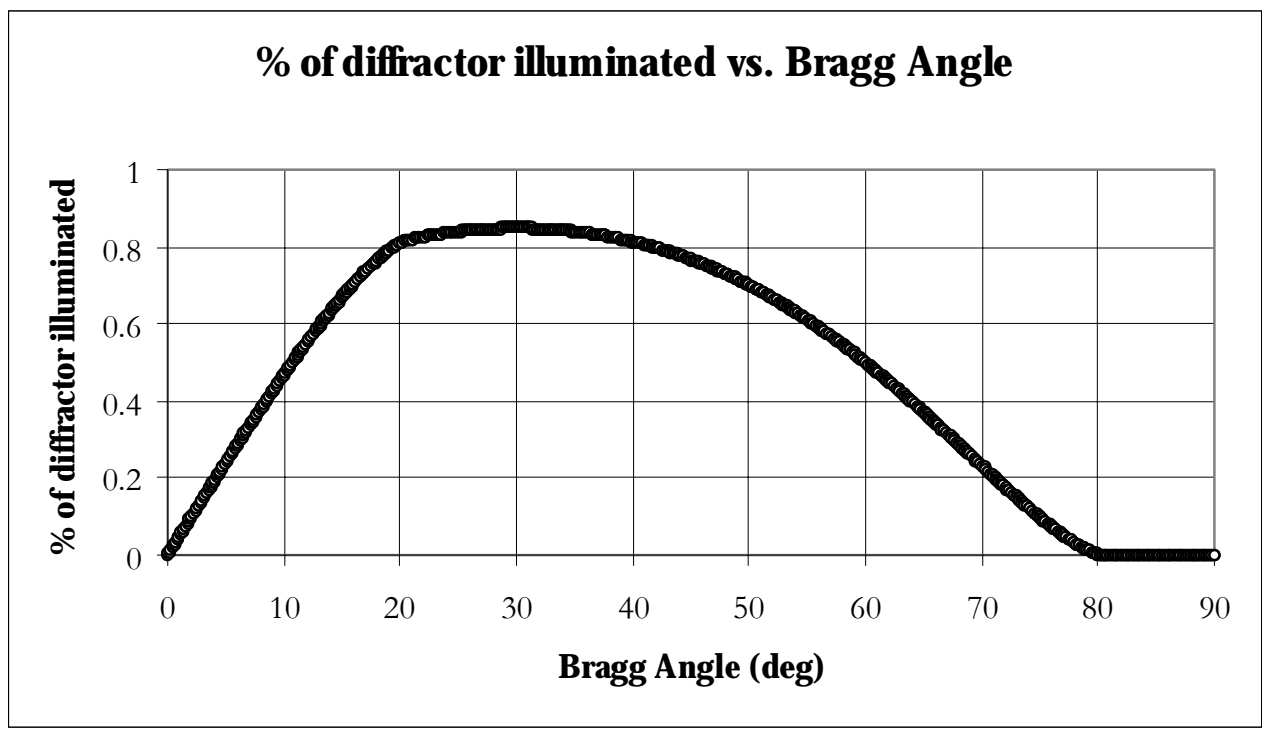

Fig. 7. Fraction of x-rays that hit diffractor for 6 sided turret. 
During Phase I, we changed our design for the Phase II spectrometer from a LEXS-like system to a de-coupled system where the diffractor turret and the detector are driven independently. This was done because we realized that the LEXS design where the detector is driven by a five-sided cam would be too slow. By eliminating the cam, the diffractor turret can be driven very rapidly and a smaller motor can be used. An advantage of a smaller motor is that it requires less power so that electrical noise is greatly reduced. The amplifier needed to drive the motor can be incorporated into the motor housing instead of requiring a separate box. A separate motor will also reduce the weight of the spectrometer box. However, we will require another motor to drive the detector and this motor will probably need to be larger than the smaller one driving the diffractor turret although it doesn't need the angular resolution of the diffractor turret motor.

Our detector must move through a total arc of 100 degrees about an axis located just below the diffractor turret. This axis of rotation is not coincident with the axis of rotation of the diffractor turret or with the face of the diffractor. The face of the diffractor rotates so neither axis is appropriate. Like LEXS, the rotation axis is chosen to minimize the displacement of the x-ray beam across the face of the detector window as the diffractor turret rotates. Some displacement of the beam is acceptable because the diameter of the detector window is larger than the diameter of the x-ray beam.

The concept of the "FasSpec" is shown in figs. 7. and 8. illustrating that both of the motor drives will be mounted to the back plane of the spectrometer. Our detector will be driven by a "timing belt" about its axis of rotation with micro-switches at each end of its angular motion to aid in determination of the zero position. The detector, its axis of rotation, and timing belt drive will be mounted to stand-offs fastened to the back plane of the spectrometer. We considered mounting them to the front plane of the spectrometer so that when the front cover is removed the detector comes free with the front plate and the diffractor turret would remain on the back plate. However, optical alignment where we use visible light to simulate the $\mathrm{x}$-ray optical path is necessary for good performance and unless we can observe the visible light on the detector window we cannot do optical alignment.

To cover the entire energy range from $100 \mathrm{eV}$ to $6500 \mathrm{eV}$ will require 6 diffractors instead of the five used on the LEXS. We will need a $2 d=200$ for Be and $B$, a $2 \mathrm{~d}=80$ for $\mathrm{N}, \mathrm{C}$, and $\mathrm{Sc}$, a $2 \mathrm{~d}=45$ for energies between $500 \mathrm{eV}$ and $1000 \mathrm{eV}$, a TAP $(2 \mathrm{~d}=25.75)$ for energies from $1000 \mathrm{eV}$ to $2000 \mathrm{eV}$, a PET $(2 \mathrm{~d}=8.79)$ for energies from $1700 \mathrm{eV}$ to $3000 \mathrm{eV}$, and either graphite or LiF for higher energies. TAP overlaps the region covered by PET and could completely replace PET in some cases but PET has sufficient resolution to separate the $\mathrm{Ta} / \mathrm{Si} / \mathrm{W}$ overlaps and has much better $\mathrm{P} / \mathrm{B}$ than $\mathrm{TAP}$ so we think that it must be used for best performance. 


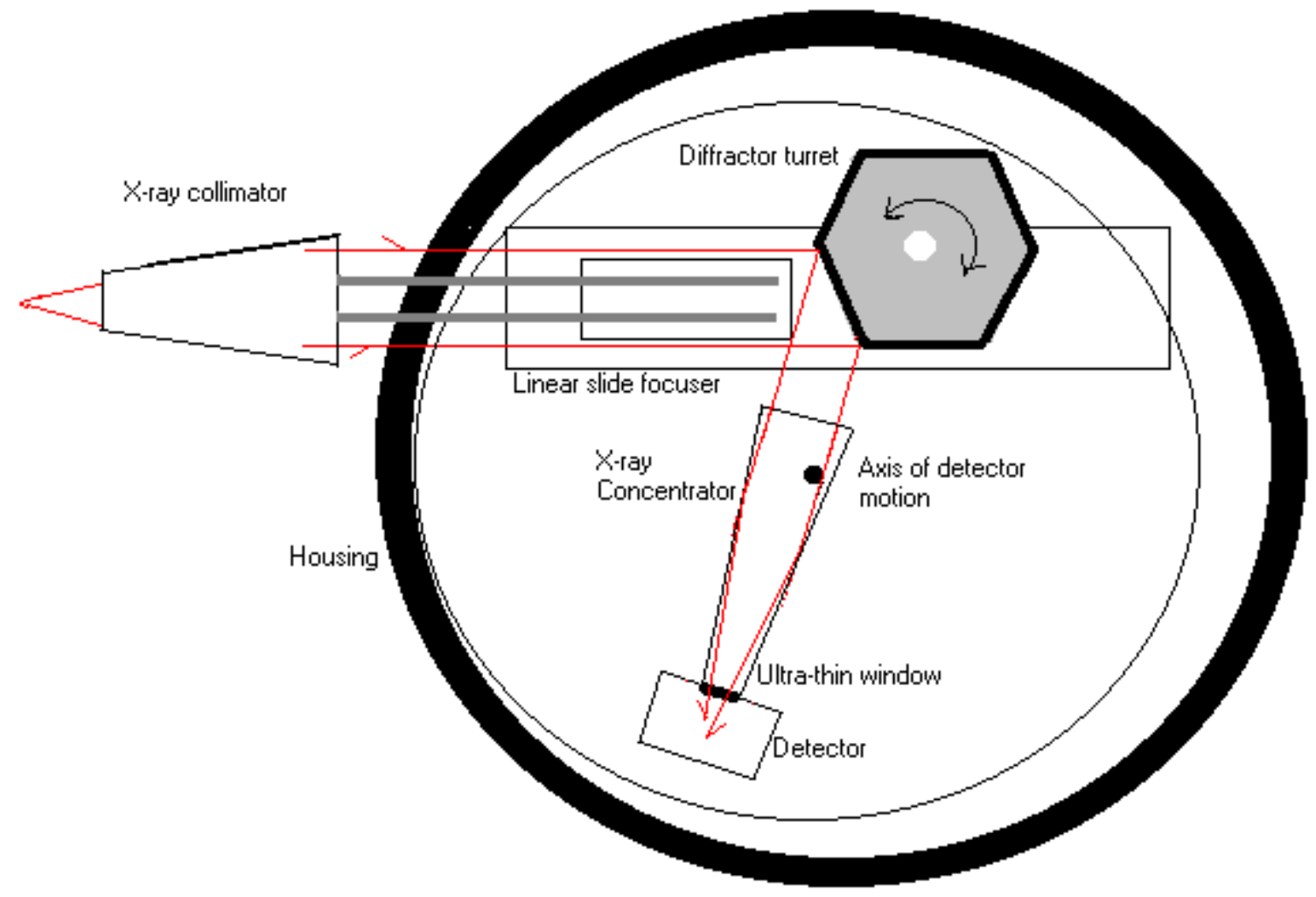

Fig. 7. Conceptual top view of FasSpec showing hexagonal diffractor turret, x-ray collimator, $\mathrm{x}$-ray concentrator and x-ray detector. 


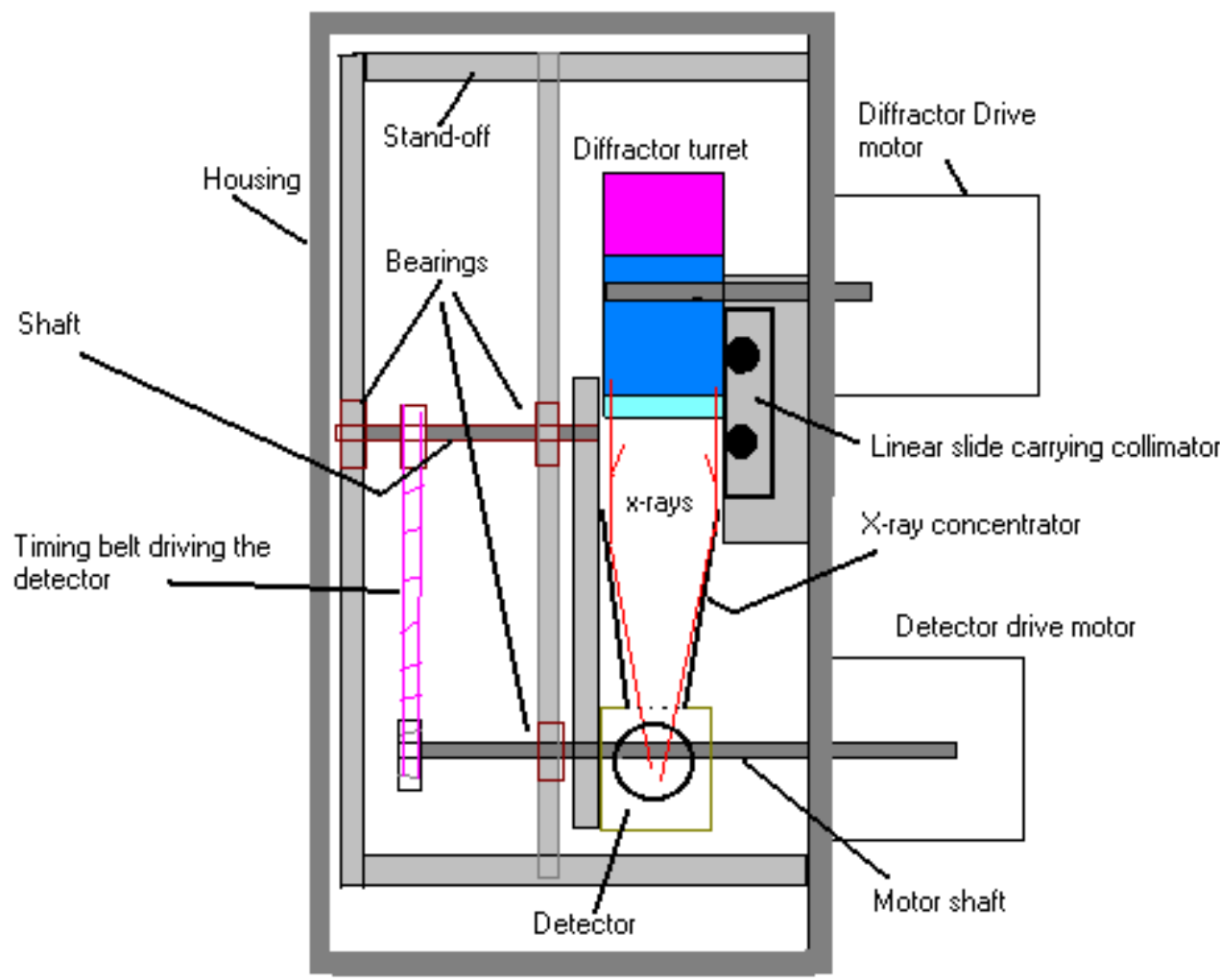

Fig. 8. Conceptual end view of FasSpec showing the drive system, diffractor turret and detector.

For this spectrometer, the hexagonal diffractor turret will have a larger diameter than the pentagonal turret used on LEXS. It is necessary for our diffractors to have a total length of $55 \mathrm{~mm}$ so they do not miss much radiation at small Bragg angles and it is this length that determines the size of the diffractor turret. The diameter of the circle swept out by the pentagonal LEXS turret is 3.4" while that swept out by the hexagonal turret will be 4.25"; a difference of .85". This small difference will not make it necessary to use a larger housing than we already use for LEXS thus preserving the small footprint that people seem to like.

To further reduce the size, weight and cost, we will build the FasSpec with a cylindrical geometry unlike the rectangular geometry of the LEXS. This will eliminate wasted space in the corners of the spectrometer making it faster to pump down to good vacuum. Use of cylindrical geometry will also reduce fabrication costs since we can buy thick walled Al pipe with 9" ID being .5" thick for the housing. We will not have to remove the central portion of a square billet to make the spectrometer housing as we do for LEXS thus saving an expensive machining step. Furthermore, it is easier to cut an oring groove in a cylindrical housing than in the square LEXS housing.

For the "FasSpec", we intend to use a smaller proportional counter detector in combination with an X-ray concentrating optic and we expect that this will be a sealed counter rather than the existing flow proportional counter used on LEXS. The reason for the smaller proportional counter is that this reduces the window area thus minimizing 
concerns about argon leakage in Field Emitter SEMs. To use a smaller window, we must use an x-ray concentrating optic in front of the counter to reduce the apparent size of he $\mathrm{x}$-ray beam from an $18 \mathrm{~mm}$ diameter to a diameter of $11.28 \mathrm{~mm}\left(100 \mathrm{~mm}^{2}\right)$. This concentrating optic is fairly simple because it does need the precise shape of the collimation optics, in fact, it can be a simple cone with entrance aperture of $20 \mathrm{~mm}$ and exit aperture of $11.28 \mathrm{~mm}$. This optic only reflects the low energy x-rays that have initially reflected from the outermost cone of the collimator while higher energy x-rays pass through its center without being altered in direction thus minimizing reflective losses for the higher energies where we need high throughput. A sealed counter using the very thin $100 \mathrm{~mm} 2$ Moxtek window should not require the inlet and outlet hoses for gas flow thus greatly simplifying the spectrometer and reducing costs of the system. Eliminating these hoses will eliminate several sources of potential leaks when the system is used in high vacuum environments.

Significant advances in data collection electronics will occur to improve performance, reduce costs and eliminate external electronic boxes. Much of this is currently being done via collaboration with EDAX but has never been incorporated into the LEXS. On LEXS, we use off-the-shelf electronics from Ortec and several of these are mounted in a NIM bin with long cables that contribute to electrical noise. We will replace the existing NIM mounted HV detector power supply from Ortec with a programmable HV module that will be mounted directly on the spectrometer. This will finally allow detector high voltage to be varied as a function of $\mathrm{x}$-ray energy without external cabling going from the HV supply to the computer. The module will also incorporate a vacuum interlock that turns off the HV when the vacuum gets near atmospheric pressure to eliminate corona between components in vacuum at mid vacuum levels. Furthermore, this is a safety issue because we currently do not have any way to turn off the HV to LEXS at atmospheric pressure when somebody wishes to remove the spectrometer cover. Lastly, this new module will eliminate the cable that goes from the HV supply to the detector thus simplifying the layout.

On the LEXS spectrometer, we use individual electronic components such as an Ortec pre-amp, amp, SCA all mounted in a NIM bin and interconnected with BNC cables. The new Digital Pulse Processor (DPP) will replace all of this and will replace an expensive counter card in the computer housing. This will completely eliminate the NIM bin and all the associated cabling thus reducing costs and sources of electrical noise.

\section{Preparation for Phase II}

Between the end of Phase I and the beginning of Phase II, we have continued working on the project with most effort being concentrated on making the optical diamond turning machine for making the mandrels for the two-reflection optics. This will be an unusual diamond turning machine because it holds both ends of the pieces being turned to allow us to accurately cut a very long and thin piece without it deflecting. The apparatus that is mounted on a granite slab for rigidity is shown partially assembled in fig.9. We expect that by the end of September 2003, that it will be doing its first cutting. 
